\title{
Drude Polarizable Force Field Parametrization of Carboxylate and N-acetyl Amine Carbohydrate Derivatives
}

Poonam Pandey ${ }^{\mathrm{a}}$, Asaminew H. Aytenfisu ${ }^{\mathrm{b}}$, Alexander D. MacKerell, Jr. ${ }^{\mathrm{b} *}$ and Sairam S. Mallajosyula $^{\mathrm{c} *}$

aDepartment of Biological Engineering, Indian Institute of Technology Gandhinagar, Simkheda, Gandhinagar, Gujarat, India

${ }^{b}$ Department of Pharmaceutical Sciences, University of Maryland School of Pharmacy, 20 Penn St., HSF II-629, Baltimore, Maryland 21201, United States

'Department of Chemistry, Indian Institute of Technology Gandhinagar, Simkheda, Gandhinagar, Gujarat, India

E-mail: alex@outerbanks.umaryland.edu, msairam@iitgn.ac.in Phone: +91 - 79 - 32454998. Fax: +91 - 79 - 23972324

\section{Supporting Material S1:}

NMR structure simulation. The lowest energy conformation of CN6 (PDB ID: 2KQO ${ }^{1}$ ) has been taken as the starting coordinates for the MD simulation study. The CN6 molecule from the additive $\mathrm{C} 36$ force field was placed in a cubic box of $\mathrm{TIP} 3 \mathrm{P}^{2}$ water with a minimum distance of $10 \AA$ from the molecule non-hydrogen atoms and the water molecules overlapping with the molecule were discarded. Three potassium ions were added to make the system neutral. To initiate system equilibration, the system was minimized using the steepest- 
descent (SD) algorithm for 200 steps followed by the adopted-basis Newton-Raphson (ABNR) method for 200 steps. Further, a 100 ps NVT simulation at $298 \mathrm{~K}$ was run to equilibrate the water molecules around the CN6 molecule, which was further followed by 200 ps NPT simulation at 1 ATM and $298 \mathrm{~K}$. The equilibrated system contained 3833 water molecules in a box with dimensions of approximately $49 \AA$ x $49 \AA$ x $49 \AA$.

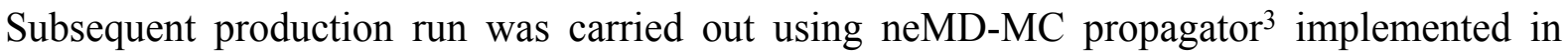
$\mathrm{NAMD}^{4}$ for $60 \mathrm{~ns}$. The integration time step was $2 \mathrm{fs}$ and the atomic coordinates were stored at each 26 ps time step. Long-range electrostatic interactions were treated with particle mesh Ewald (PME) summation method ${ }^{5}$ with a real-space cutoff value $12 \AA$ and the neighbor list were updated within $14 \AA$. Non-bonded van der Waals interactions were truncated with a cutoff between $10 \AA$ and $12 \AA$ using a switching function. Temperature was maintained at $298 \mathrm{~K}$ using Langevin thermostat with a damping coefficient of $5 \mathrm{ps}^{-1}$. The pressure was set to 1 ATM using the Langevin piston method ${ }^{6}$ with an oscillation period of $200 \mathrm{fs}$ and the damping time scale of 100 fs. The pressure coupling was applied isotropically and the periodic boundary conditions were applied in all the three dimensions. Water molecules were rigidified using the $\mathrm{SETTLE}^{7}$ algorithm, while all bonds involving hydrogen atoms were constrained using the $\mathrm{SHAKE}^{8}$ algorithm.

To prepare the Drude polarizable system, the equilibrated additive coordinates were converted to the Drude polarizable model in CHARMM by constructing Drude oscillators and lone pairs. The TIP3P ${ }^{2}$ water molecules were also replaced with polarizable SWM4-NDP water model ${ }^{9}$. Positions of Drude particles were relaxed by using 200 steps of SD energy minimization in CHARMM. All bonds involving hydrogen were constrained using SHAKE algorithm and the system was minimized by performing 200 steps SD, followed my 200 steps ABNR energy minimization. System was equilibrated by performing 100 ps NVT using velocity-Verlet algorithm ${ }^{10}$ with Nose-Hoover dual thermostat at $298 \mathrm{~K}$, followed by 200 ps 
NPT simulation at $298 \mathrm{~K}$ and 1 ATM pressure. The thermostat coupled to the Drude oscillator was set to $1 \mathrm{~K}$, while thermostat for the real atoms was set to $298 \mathrm{~K}$.

Subsequent production run was carried out using neMD-MC propagator ${ }^{3}$ implemented in $\mathrm{NAMD}^{4}$ for $60 \mathrm{~ns}$. The temperature for real atoms was fixed at $298 \mathrm{~K}$ using Langevin dynamics ${ }^{10}$, while the thermostat coupled to the Drude oscillator was set to $1 \mathrm{~K}$. For selected non-bonded pairs $\left(\mathrm{K}^{+}\right.$ion - carbonyl oxygen $(\mathrm{O}), \mathrm{K}^{+}$ion - carboxylate oxygen $(\mathrm{O} 61 / \mathrm{O} 62)$ and $\mathrm{K}^{+}$ion - exocyclic oxygen (O6)), pairwise screened Coulomb correction (NBTHOLE) was calculated with a cutoff value of $5 \AA$. For the carboxylate oxygen (O61/O62) and $\mathrm{K}^{+}$ ion interactions, an NBFIX value of $\varepsilon=-0.16850 \mathrm{kcal} / \mathrm{mol}$ was applied with $\mathrm{R}_{\min }=3.75 \AA$, while an NBTHOLE value of 0.75 was used (Justin Lemkul, personal communication). For the carbonyl oxygen $(\mathrm{O})$ and $\mathrm{K}^{+}$ion interactions, an NBFIX value of $\varepsilon=-0.18000 \mathrm{kcal} / \mathrm{mol}$ was applied with $\mathrm{R}_{\min }=3.21 \AA$, while an NBTHOLE value of 2.19 was used. For the exocyclic oxygen (O6) and $\mathrm{K}^{+}$ion interactions, an NBFIX value of $\varepsilon=-0.15000 \mathrm{kcal} / \mathrm{mol}$ was applied with $R_{\min }=3.34 \AA$, while an NBTHOLE value of 1.82 was used. The pressure was maintained at 1 ATM using Langevin piston ${ }^{6}$ method. Long-range electrostatic interactions were calculated using PME with a real-space cutoff value $12 \AA$ and the neighbor list were updated within $16 \AA$. Non-bonded van der Waals interactions were truncated with a cut-off between $10 \AA$ and $12 \AA$ using a switching function. To avoid polarization catastrophe, a "hard wall constraint" was used with a Drude-atom bond length cutoff of $0.2 \AA$. The simulation trajectories obtained from production runs using the additive and Drude polarizable force fields were stored for analysis. 
Table S1. Vibrational Frequency analysis of model compound M1. QM frequencies were calculated at the MP2/6-31G* model chemistry. QM frequencies have been scaled by 0.9434 . Vibrational modes follow the internal coordinate system of Pulay et al. ${ }^{11}$

\begin{tabular}{|c|c|c|c|c|c|c|c|c|}
\hline \multicolumn{3}{|c|}{ QM } & \multicolumn{3}{|c|}{ Additive } & \multicolumn{3}{|c|}{ Drude } \\
\hline Frequency & $\begin{array}{l}\text { vibrational } \\
\text { mode }^{a}\end{array}$ & contribution & Frequency & $\begin{array}{l}\text { vibrational } \\
\text { mode }\end{array}$ & contribution & Frequency & $\begin{array}{l}\text { vibrational } \\
\text { mode }\end{array}$ & contribution \\
\hline \multirow[t]{2}{*}{44.9} & tor1 & 99 & 60.8 & tor1 & 87 & 39.5 & tor1 & 100 \\
\hline & & & & tor2 & 16 & & & \\
\hline \multirow[t]{2}{*}{59} & tor2 & 79 & 70.3 & tor2 & 76 & 95.9 & tor2 & 86 \\
\hline & & & & tor1 & 17 & & & \\
\hline \multirow[t]{2}{*}{197.7} & tor3 & 55 & 194.5 & tor3 & 65 & 204.9 & yczC1 & 49 \\
\hline & & & & $x c y C 1$ & 15 & & tor3 & 39 \\
\hline \multirow[t]{3}{*}{208.3} & $\mathrm{xcyC1}$ & 45 & 243.6 & tor4 & 56 & 220.7 & tor4 & 44 \\
\hline & roCOO & 21 & & xсyC1 & 15 & & tor3 & 26 \\
\hline & tor3 & 16 & & & & & & \\
\hline \multirow[t]{2}{*}{221.3} & tor4 & 72 & 251.1 & xсyC1 & 37 & 230.1 & xcyC1 & 56 \\
\hline & & & & tor4 & 28 & & roCOO & 17 \\
\hline \multirow[t]{2}{*}{299.2} & yczC1 & 38 & 300.1 & yczC1 & 52 & 307.6 & $\mathrm{sC1}-\mathrm{C} 2$ & 33 \\
\hline & tor4 & 19 & & & & & tor4 & 22 \\
\hline \multirow[t]{2}{*}{358} & ang1 & 45 & 346.1 & $\mathrm{xczC1}$ & 61 & 322.4 & roCOO & 36 \\
\hline & $\mathrm{sC} 1-\mathrm{C} 2$ & 27 & & & & & $\mathrm{xczC1}$ & 16 \\
\hline \multirow[t]{2}{*}{395.4} & $\mathrm{xczC1}$ & 50 & 410 & ang1 & 33 & 370 & $\mathrm{xczC1}$ & 44 \\
\hline & rocOO & 15 & & $\mathrm{sC} 1-\mathrm{C} 2$ & 18 & & ang1 & 19 \\
\hline \multirow[t]{3}{*}{467.8} & roCOO & 42 & 483 & roCOO & 60 & 431.3 & roCOO & 28 \\
\hline & $\mathrm{xczC1}$ & 16 & & ang1 & 16 & & ang1 & 26 \\
\hline & & & & & & & $x c z C 1$ & 17 \\
\hline \multirow[t]{2}{*}{634.6} & sdefCOO & 32 & 662.3 & wagCOO & 49 & 545.1 & wagCOO & 81 \\
\hline & wagCOO & 18 & & sdefCOO & 18 & & & \\
\hline \multirow[t]{3}{*}{725.1} & wagCOO & 54 & 754.4 & sdefCOO & 41 & 657.1 & ang1 & 21 \\
\hline & sdefCOO & 16 & & wagCOO & 29 & & sdefCOO & 20 \\
\hline & & & & & & & $\mathrm{sC} 1-\mathrm{C} 2$ & 19 \\
\hline \multirow[t]{2}{*}{798.7} & sdefCOO & 19 & 859.6 & ropMe0 & 17 & 815.5 & sC1-OM & 43 \\
\hline & ropMe0 & 15 & & sC1-C2 & 16 & & & \\
\hline \multirow[t]{3}{*}{857.4} & roMe0 & 26 & 933.3 & roMe0 & 46 & 884.1 & sdefCOO & 52 \\
\hline & sC1-OM & 25 & & & & & $\mathrm{sC2}-\mathrm{O}$ & 17 \\
\hline & $\mathrm{sC} 1-\mathrm{C} 2$ & 21 & & & & & $\mathrm{sC1}-\mathrm{C} 2$ & 16 \\
\hline \multirow[t]{3}{*}{1005.5} & sC1-C0 & 24 & 1029 & ropMe0 & 51 & 1010.2 & ropMe0 & 57 \\
\hline & adefC1 & 21 & & & & & & \\
\hline & ropMe0 & 15 & & & & & & \\
\hline 1051.8 & sOM-CM & 32 & 1034 & sOM-CM & 59 & 1032.8 & sOM-CM & 53 \\
\hline & ropMe0 & 21 & & & & & roMe0 & 26 \\
\hline 1087.8 & $\mathrm{sC} 1-\mathrm{CO}$ & 29 & 1050.5 & sC1-C0 & 48 & 1038.6 & sC1-C0 & 63 \\
\hline & roMe0 & 26 & & roMe0 & 17 & & & \\
\hline 1107.2 & sOM-CM & 42 & 1113.3 & roMeM & 46 & 1069.7 & roMe0 & 39 \\
\hline & sC1-OM & 23 & & & & & sOM-CM & 17 \\
\hline 1140.2 & roMeM & 71 & 1135.2 & ropMeM & 44 & 1128.7 & roMeM & 43 \\
\hline & ropMeM & 23 & & roMeM & 22 & & ropMeM & 37 \\
\hline & & & & & & & adefpMeM & 19 \\
\hline 1174.5 & ropMeM & 51 & 1221.3 & sC1-OM & 27 & 1163.5 & ropMeM & 37 \\
\hline & roMeM & 18 & & sdefC1 & 18 & & roMeM & 29 \\
\hline & & & & ropMeM & 18 & & & \\
\hline 1258.4 & adefC1 & 56 & 1309.3 & adefC1 & 46 & 1283.4 & $\mathrm{sC2}-\mathrm{O}$ & 74 \\
\hline & $\mathrm{sC2}-\mathrm{O}$ & 19 & & $\mathrm{sC2}-\mathrm{O}$ & 19 & & & \\
\hline 1306.2 & $\mathrm{sC2}-\mathrm{O}$ & 47 & 1377 & $\mathrm{sC} 2-\mathrm{O}$ & 33 & 1310.9 & adefC1 & 65 \\
\hline & & & & sdefMe0 & 17 & & & \\
\hline & & & & $\mathrm{sC1}-\mathrm{C} 2$ & 16 & & & \\
\hline 1316.8 & sdefC1 & 84 & 1399 & sdefMe0 & 69 & 1407.5 & sdefMe0 & 78 \\
\hline 1352.9 & sdefMe0 & 65 & 1433.7 & adefMe0 & 48 & 1423.4 & adefMe0 & 60 \\
\hline & & & & adefpMe0 & 43 & & adefpMe0 & 28 \\
\hline 1435.4 & sdefMeM & 83 & 1440.4 & adefMeM & 79 & 1436.5 & adefMeM & 73 \\
\hline
\end{tabular}




\begin{tabular}{|l|l|l|l|l|l|l|l|l|}
\hline & & & & & & ropMeM & 15 \\
\hline 1459 & adefpMeM & 67 & 1446.1 & adefpMe0 & 40 & 1451.6 & adefpMeM & 33 \\
\hline & adefpMe0 & 15 & & adefMe0 & 33 & & adefpMe0 & 26 \\
\hline 1469.2 & adefpMe0 & 64 & 1461.6 & adefpMeM & 68 & 1469.9 & sdefC1 & 40 \\
\hline & adefMe0 & 19 & & & & & adefpMe0 & 20 \\
\hline 1476.1 & adefMe0 & 56 & 1557 & sC2-O & 35 & 1479.7 & adefpMeM & 33 \\
\hline & adefpMeM & 16 & & sdefC1 & 30 & & sdefC1 & 22 \\
\hline & & & & sdefMeM & 17 & & & \\
\hline 1489.5 & adefMeM & 80 & 1594.5 & sdefMeM & 66 & 1594.4 & sdefMeM & 85 \\
\hline & & & & sC2-O & 18 & & & \\
\hline 1689.3 & sC2-O & 95 & 1636.7 & sC2-O & 41 & 1635.7 & sC2-O & 87 \\
\hline & & & & sdefC1 & 28 & & & \\
\hline 2842.2 & sC1-H1 & 52 & 2849.6 & sCM-HM & 99 & 2849.3 & sCM-HM & 100 \\
\hline & sCM-HM & 48 & & & & & & \\
\hline 2849.6 & sCM-HM & 52 & 2858.8 & sC1-H1 & 98 & 2897.9 & sC0-H0 & 59 \\
\hline 2894.3 & sC1-H1 & 47 & & & & & sC1-H1 & 40 \\
\hline & sCM-HM & 86 & 2901.5 & sCO-H0 & 99 & 2905.6 & sC1-H1 & 58 \\
\hline 2910.8 & sC0-H0 & 86 & 2913.6 & sCM-HM & 99 & 2913.2 & sCO-H0 & 40 \\
\hline 2974.2 & sCM-HM & 99 & 2915.2 & sCM-HM & 99 & 2914.5 & sCM-HM & 99 \\
\hline 2984.3 & sCO-H0 & 98 & 2960 & sCO-H0 & 100 & 2957.3 & sCO-H0 & 100 \\
\hline 3041.4 & sCO-HO & 100 & 2960.7 & sCO-H0 & 100 & 2959.6 & sCO-HO & 99 \\
\hline
\end{tabular}

a tor1=OM-C1-C2-O9, tor2= C0C1OMCM, tor3= C1OMCMHM, tor4= OMC1C0H0; ang1= $\mathrm{C} 1 \mathrm{OMCM}$; ro=rocking; s=stretching; sdef=symmetric deformation; adef=asymmetric deformation; wag=wagging 
Table S2. Vibrational Frequency analysis of model compound M2. QM frequencies were calculated at the MP2/6-31G* model chemistry. QM frequencies have been scaled by 0.9434 . Vibrational modes follow the internal coordinate system of Pulay et al. ${ }^{11}$

\begin{tabular}{|c|c|c|c|c|c|c|c|c|}
\hline \multicolumn{3}{|c|}{ QM } & \multicolumn{3}{|c|}{ Additive } & \multicolumn{3}{|c|}{ Drude } \\
\hline Frequency & $\begin{array}{l}\text { vibrational } \\
\text { mode }\end{array}$ & contribution & Frequency & $\begin{array}{l}\text { vibrational } \\
\text { mode }\end{array}$ & contribution & Frequency & $\begin{array}{l}\text { vibrational } \\
\text { mode }\end{array}$ & contribution \\
\hline \multirow[t]{2}{*}{51.7} & tor1 & 86 & 60.9 & tor1 & 65 & 64.4 & tor1 & 75 \\
\hline & tor2 & 17 & tor2 & 34 & & & tor3 & 20 \\
\hline \multirow[t]{2}{*}{60.1} & tor2 & 62 & 73.1 & tor2 & 57 & 65.5 & tor3 & 79 \\
\hline & tor3 & 28 & tor1 & 35 & & & & \\
\hline \multirow[t]{2}{*}{94.2} & tor3 & 63 & 129.7 & tor3 & 78 & 85.1 & tor2 & 88 \\
\hline & tor2 & 18 & & & & & tor1 & 17 \\
\hline \multirow[t]{2}{*}{178.1} & defCNCr & 53 & 204.7 & defCNCr & 49 & 206.9 & defCNCr & 57 \\
\hline & roCO & 21 & roCO & 21 & & & defNCrC & 18 \\
\hline \multirow[t]{2}{*}{240.3} & tor4 & 60 & 257.3 & tor5 & 53 & 254 & tor5 & 60 \\
\hline & tor5 & 24 & tor4 & 32 & & & tor4 & 24 \\
\hline \multirow[t]{2}{*}{272.7} & tor5 & 55 & 269.6 & tor4 & 55 & 265.5 & tor4 & 63 \\
\hline & tor4 & 25 & tor5 & 29 & & & tor5 & 22 \\
\hline \multirow[t]{2}{*}{303.6} & defCCrC & 27 & 299.2 & defNCrC & 30 & 317.6 & defNCrC & 36 \\
\hline & defNCrC & 24 & defCCrC & 23 & & & defCCrC & 27 \\
\hline \multirow[t]{2}{*}{360.1} & wagNH & 88 & 385 & defNCrC & 48 & 373 & defNCrC & 56 \\
\hline & & & roCO & 16 & & & wagNH & 20 \\
\hline 393.8 & defNCrC & 60 & 422 & defCCrC & 46 & 421.2 & defCCrC & 50 \\
\hline \multirow[t]{3}{*}{411.5} & defClCN & 36 & 490.3 & defNCrC & 39 & 526.6 & wagNH & 34 \\
\hline & defCCrC & 29 & defCICN & 25 & & & & \\
\hline & & & roCO & 17 & & & roCO & 18 \\
\hline \multirow[t]{3}{*}{456.1} & defNCrC & 46 & 584.6 & defClCN & 33 & 566.5 & defNCrC & 23 \\
\hline & & & $\mathrm{sCl}-\mathrm{C}$ & 28 & & & wagNH & 19 \\
\hline & & & roCO & 15 & & & roco & 16 \\
\hline \multirow[t]{3}{*}{574.7} & wagCO & 33 & 655.2 & wagCO & 70 & 629.5 & defCICN & 49 \\
\hline & $\mathrm{sCl}-\mathrm{C}$ & 18 & wagNH & 26 & & & $\mathrm{sCl}-\mathrm{C}$ & 31 \\
\hline & defCICN & 16 & & & & & & \\
\hline \multirow[t]{2}{*}{612.7} & wagCO & 47 & 813.6 & wagNH & 37 & 797.4 & wagCO & 49 \\
\hline & & & sCR-C2 & 17 & & & wagNH & 15 \\
\hline \multirow[t]{2}{*}{829.1} & sCR-C2 & 52 & 824 & sC-N & 22 & 825.2 & $\mathrm{sCl}-\mathrm{C}$ & 37 \\
\hline & roMer & 16 & wagNH & 21 & & & sCR-C2 & 17 \\
\hline 889.2 & ropMer & 21 & 850 & sCR-C2 & 30 & 860.4 & sCR-C2 & 21 \\
\hline & sC-N & 18 & roMer & 22 & & & roMer & 18 \\
\hline 905.9 & roMer & 53 & 926 & ropMer & 46 & 928.8 & ropMer & 39 \\
\hline & & & roMer & 15 & & & roMer & 21 \\
\hline 937.4 & ropMer & 39 & 942.4 & roMer & 46 & 949.2 & ropMer & 47 \\
\hline & sCR-C2 & 35 & ropMer & 28 & & & roMer & 32 \\
\hline & roMer & 16 & sCR-C2 & 18 & & & sCR-C2 & 17 \\
\hline 956.8 & ropMe & 32 & 960.3 & ropMer & 44 & 967.4 & roMer & 49 \\
\hline & $\mathrm{sCl}-\mathrm{C}$ & 27 & roMer & 39 & & & ropMer & 29 \\
\hline 1007.7 & ropMe & 34 & 979.1 & ropMe & 55 & 1031 & ropMe & 49 \\
\hline & ropMer & 18 & roMe & 20 & & & & \\
\hline 1025 & roMe & 73 & 1078.9 & roMe & 38 & 1080 & roMe & 46 \\
\hline & wagCO & 19 & wagCO & 16 & & & wagCO & 17 \\
\hline & & & ropMe & 16 & & & & \\
\hline 1130.6 & sCR-C2 & 37 & 1096.6 & roMer & 25 & 1097.6 & roMer & 20 \\
\hline & ropMer & 21 & & & & & & \\
\hline & defNCrC & 17 & & & & & & \\
\hline 1154.1 & roMer & 23 & 1123.2 & sCR-C2 & 37 & 1118.5 & sCR-C2 & 28 \\
\hline & & & $\mathrm{ropCrH}$ & 29 & & & ropCrH & 25 \\
\hline 1175.7 & $\mathrm{sN}-\mathrm{CR}$ & 29 & 1158.3 & $\mathrm{sN}-\mathrm{CR}$ & 30 & 1135.7 & sCR-C2 & 21 \\
\hline & & & $\mathrm{roCrH}$ & 16 & & & $\mathrm{roCrH}$ & 18 \\
\hline & & & & & & & $\mathrm{sN}-\mathrm{CR}$ & 15 \\
\hline 1231.3 & roNH & 33 & 1268.5 & roNH & 33 & 1160.8 & roNH & 23 \\
\hline & sC-N & 19 & $\mathrm{sCl}-\mathrm{C}$ & 22 & & & sC-N & 18 \\
\hline 1312.6 & ropCrH & 30 & 1343.9 & ropCrH & 60 & 1338.2 & ropCrH & 60 \\
\hline
\end{tabular}




\begin{tabular}{|c|c|c|c|c|c|c|c|c|}
\hline & $\mathrm{roCrH}$ & 28 & sCR-C2 & 16 & & & sCR-C2 & 19 \\
\hline \multirow[t]{2}{*}{1343.9} & $\mathrm{roCrH}$ & 36 & 1384.1 & sdefMe & 91 & 1387.8 & roCrH & 53 \\
\hline & ropCrH & 35 & & & & & roNH & 17 \\
\hline 1369.9 & sdefMe & 71 & 1395 & sdefMer & 90 & 1404 & sdefMer & 94 \\
\hline 1371.1 & sdefMer & 77 & 1397.9 & sdefMer & 79 & 1408.5 & sdefMer & 84 \\
\hline \multirow[t]{2}{*}{1388.8} & sdefMer & 94 & 1408.4 & adefpMe & 34 & 1420.6 & adefMe & 83 \\
\hline & & & $\mathrm{roCrH}$ & 22 & & & & \\
\hline \multirow[t]{2}{*}{1444.6} & adefpMe & 53 & 1418.5 & adefMe & 38 & 1426.5 & adefMer & 93 \\
\hline & adefMe & 30 & $\mathrm{roCrH}$ & 18 & & & & \\
\hline \multirow[t]{3}{*}{1458} & adefpMer & 31 & 1418.6 & adefpMe & 44 & 1431.8 & adefMer & 84 \\
\hline & adefpMe & 26 & adefMe & 34 & & & & \\
\hline & adefMer & 22 & & & & & & \\
\hline \multirow[t]{2}{*}{1461.6} & adefMe & 35 & 1429.5 & adefMer & 81 & 1432.9 & adefpMe & 86 \\
\hline & adefMer & 21 & & & & & & \\
\hline \multirow[t]{2}{*}{1464.8} & adefpMer & 51 & 1432.1 & adefpMer & 72 & 1435.5 & adefpMer & 84 \\
\hline & adefMer & 42 & adefMer & 18 & & & & \\
\hline \multirow[t]{2}{*}{1469.9} & adefpMer & 51 & 1434.9 & adefMer & 80 & 1439 & adefpMer & 83 \\
\hline & adefMer & 21 & & & & & & \\
\hline 1483.9 & adefMer & 83 & 1437.5 & adefpMer & 76 & 1459.1 & sdefMe & 98 \\
\hline \multirow[t]{4}{*}{1492.2} & roNH & 35 & 1583.4 & roNH & 26 & 1595.5 & roNH & 44 \\
\hline & sC-N & 18 & $\mathrm{roCrH}$ & 20 & & & $s N-C R$ & 21 \\
\hline & adefpMer & 16 & sC-N & 18 & & & & \\
\hline & & & $\mathrm{sN}-\mathrm{CR}$ & 17 & & & & \\
\hline 1687.2 & sC-O & 79 & 1683.8 & sC-O & 66 & 1725.9 & sC-O & 70 \\
\hline \multirow[t]{3}{*}{2921.4} & $\mathrm{sC} 22-\mathrm{H}$ & 92 & 2898.8 & $\mathrm{sC} 21-\mathrm{H}$ & 41 & 2846.9 & $\mathrm{sCl}-\mathrm{H}$ & 100 \\
\hline & & & $\mathrm{SC} 22-\mathrm{H}$ & 39 & & & & \\
\hline & & & SCR-HR & 20 & & & & \\
\hline \multirow[t]{3}{*}{2925} & $\mathrm{sC21-H}$ & 92 & 2900.3 & $\mathrm{sC} 22-\mathrm{H}$ & 52 & 2896.6 & SCR-HR & 50 \\
\hline & & & $\mathrm{sC} 21-\mathrm{H}$ & 48 & & & $\mathrm{sC21-H}$ & 26 \\
\hline & & & & & & & $\mathrm{sC} 22-\mathrm{H}$ & 24 \\
\hline \multirow[t]{2}{*}{2938.7} & $\mathrm{sCl}-\mathrm{H}$ & 100 & 2909.7 & sCR-HR & 78 & 2902 & $\mathrm{sC} 22-\mathrm{H}$ & 51 \\
\hline & & & & & & & $\mathrm{sC} 21-\mathrm{H}$ & 49 \\
\hline \multirow[t]{3}{*}{2965.2} & SCR-HR & 92 & 2917 & $\mathrm{sCl}-\mathrm{H}$ & 100 & 2907.6 & sCR-HR & 49 \\
\hline & & & & & & & $\mathrm{sC} 21-\mathrm{H}$ & 26 \\
\hline & & & & & & & $\mathrm{sC} 22-\mathrm{H}$ & 25 \\
\hline \multirow[t]{2}{*}{3002.6} & $\mathrm{sC} 22-\mathrm{H}$ & 65 & 2957.7 & $\mathrm{sC21-H}$ & 52 & 2917.4 & $\mathrm{sCl}-\mathrm{H}$ & 100 \\
\hline & $\mathrm{sC21-H}$ & 35 & $\mathrm{SC} 22-\mathrm{H}$ & 48 & & & & \\
\hline \multirow[t]{2}{*}{3009.2} & $\mathrm{sC21-H}$ & 63 & 2958.3 & $\mathrm{sC} 22-\mathrm{H}$ & 63 & 2918.2 & $\mathrm{sCl}-\mathrm{H}$ & 99 \\
\hline & $\mathrm{sC} 22-\mathrm{H}$ & 31 & $\mathrm{sC} 21-\mathrm{H}$ & 37 & & & & \\
\hline \multirow[t]{2}{*}{3020.2} & $\mathrm{sCl}-\mathrm{H}$ & 100 & 2959.5 & $\mathrm{sC} 21-\mathrm{H}$ & 64 & 2957.8 & $\mathrm{sC} 22-\mathrm{H}$ & 63 \\
\hline & & & $\mathrm{SC} 22-\mathrm{H}$ & 36 & & & $\mathrm{sC} 21-\mathrm{H}$ & 37 \\
\hline \multirow[t]{2}{*}{3024.2} & $\mathrm{sC} 22-\mathrm{H}$ & 99 & 2960.5 & $\mathrm{sC} 22-\mathrm{H}$ & 52 & 2959.2 & $\mathrm{sC} 21-\mathrm{H}$ & 62 \\
\hline & & & $\mathrm{sC} 21-\mathrm{H}$ & 46 & & & $\mathrm{sC} 22-\mathrm{H}$ & 38 \\
\hline \multirow[t]{2}{*}{3031.4} & $\mathrm{sC21-H}$ & 100 & 2975.2 & $\mathrm{sCl}-\mathrm{H}$ & 100 & 2960.1 & $\mathrm{sC} 21-\mathrm{H}$ & 57 \\
\hline & & & & & & & $\mathrm{sC} 22-\mathrm{H}$ & 43 \\
\hline \multirow[t]{2}{*}{3047.7} & $\mathrm{sCl}-\mathrm{H}$ & 100 & 2975.3 & $\mathrm{sCl}-\mathrm{H}$ & 100 & 2962.1 & $\mathrm{sC} 22-\mathrm{H}$ & 55 \\
\hline & & & & & & & $\mathrm{sC} 21-\mathrm{H}$ & 44 \\
\hline 3435.8 & $\mathrm{sN}-\mathrm{H}$ & 100 & 3325.3 & $\mathrm{sN}-\mathrm{H}$ & 99 & 3397.3 & $\mathrm{sN}-\mathrm{H}$ & 100 \\
\hline
\end{tabular}

a tor $1=\mathrm{HCCN}$, tor $2=\mathrm{CNCC}$, tor $3=\mathrm{CCNC}$, tor $4=\mathrm{NCCH} 2$, tor $5=\mathrm{NCCH}$; ro $=$ rocking; $\mathrm{s}=$ stretching; sdef=symmetric deformation; adef=asymmetric deformation; wag=wagging 
Table S3. Classical Drude oscillator polarizable force field topologies and parameters for carboxylate and $\mathrm{N}$-acetyl amine carbohydrate derivatives.

* DRUDE topology and parameter stream file for

* Carboxylate and N-acetyl Amine Carbohydrate Derivatives

read $\mathrm{rtf}$ card append

* Drude carbohydrates

41

DEFA FIRS NONE LAST NONE

AUTOGENERATE ANGLES DIHEDRALS DRUDE PATCH

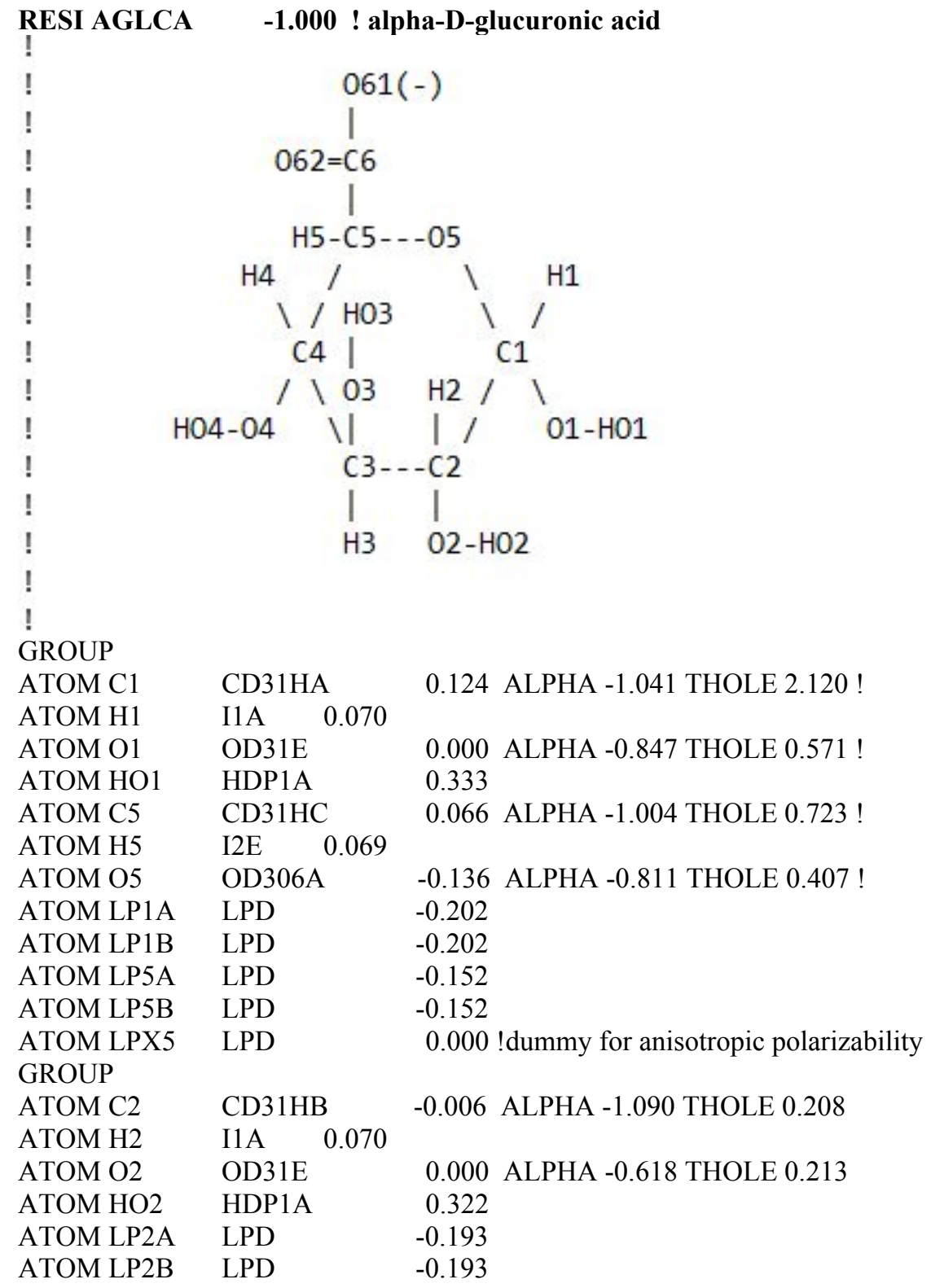




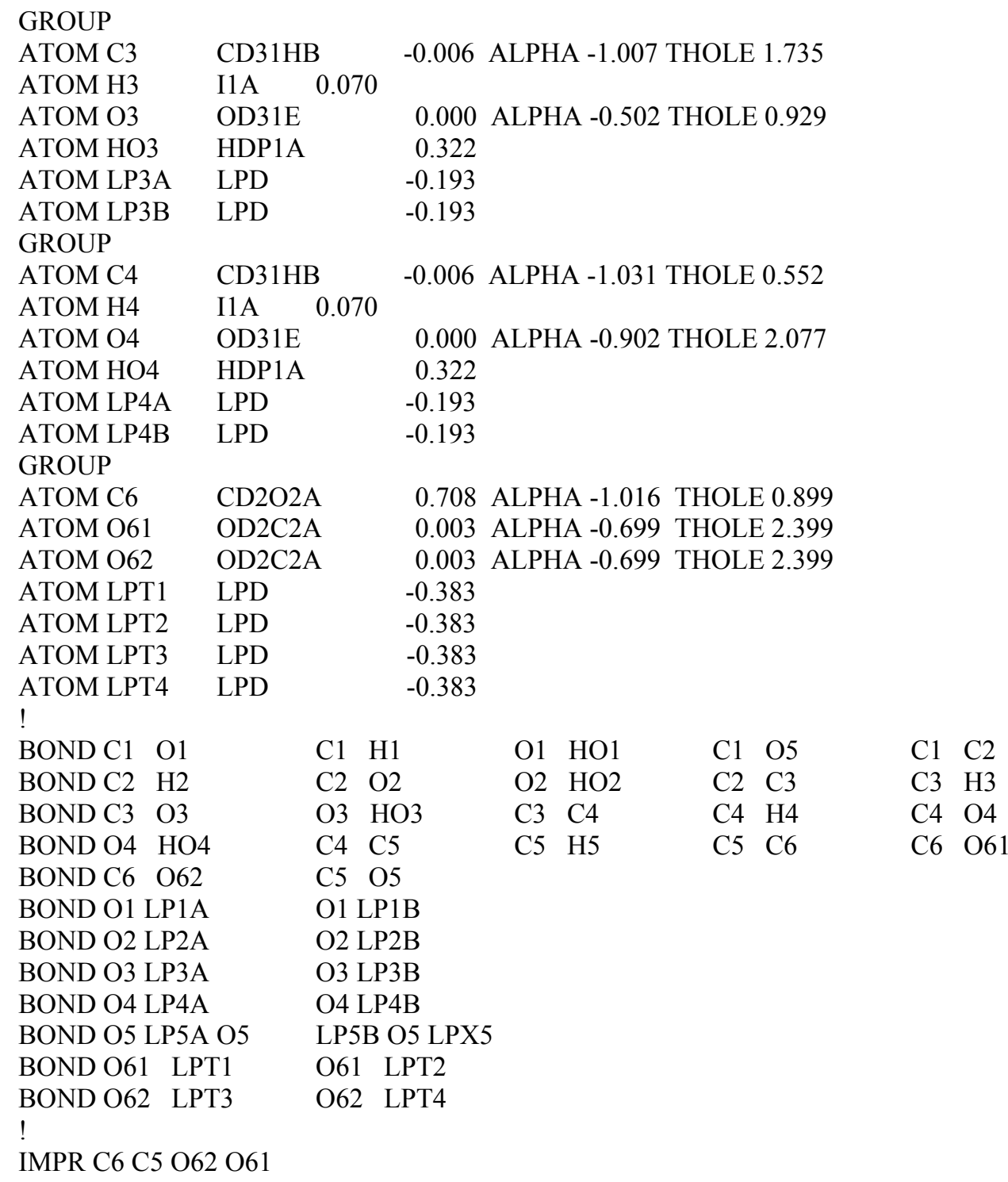

LONEPAIR relative LP1A O1 C1 HO1 distance 0.35 angle 110.0 dihe 91.0 LONEPAIR relative LP1B O1 C1 HO1 distance 0.35 angle 110.0 dihe 269.0 ANISOTROPY O1 C1 LP1A LP1B A11 0.8108 A22 1.2162

LONEPAIR relative LP2A O2 C2 HO2 distance 0.35 angle 110.0 dihe 91.0 LONEPAIR relative LP2B O2 C2 HO2 distance 0.35 angle 110.0 dihe 269.0 ANISOTROPY O2 C2 LP2A LP2B A11 0.8108 A22 1.2162

LONEPAIR relative LP3A O3 C3 HO3 distance 0.35 angle 110.0 dihe 91.0 LONEPAIR relative LP3B O3 C3 HO3 distance 0.35 angle 110.0 dihe 269.0 ANISOTROPY O3 C3 LP3A LP3B A11 0.8108 A22 1.2162

LONEPAIR relative LP4A O4 C4 HO4 distance 0.35 angle 110.0 dihe 91.0 LONEPAIR relative LP4B O4 C4 HO4 distance 0.35 angle 110.0 dihe 269.0 ANISOTROPY O4 C4 LP4A LP4B A11 0.8108 A22 1.2162

LONEPAIR bisector LP5A O5 C1 C5 distance 0.35 angle 110.0 dihe 90.0 LONEPAIR bisector LP5B O5 C1 C5 distance 0.35 angle 110.0 dihe 270.0 LONEPAIR bisector LPX5 O5 C1 C5 distance 0.10 angle 0.0 dihe 0.0 ANISOTROPY O5 LPX5 LP5A LP5B A11 0.8889 A22 1.2222

LONEPAIR relative LPT1 O61 C6 O62 distance 0.35 angle 110.0 dihe 0.0 
LONEPAIR relative LPT2 O61 C6 O62 distance 0.35 angle 110.0 dihe 180.0 LONEPAIR relative LPT3 O62 C6 O61 distance 0.35 angle 110.0 dihe 0.0 LONEPAIR relative LPT4 O62 C6 O61 distance 0.35 angle 110.0 dihe 180.0 ANISOTROPY O61 C6 LPT1 LPT2 A11 0.7229 A22 1.265

ANISOTROPY O62 C6 LPT3 LPT4 A11 0.7229 A22 1.265

\begin{tabular}{|c|c|c|c|c|c|c|c|c|c|}
\hline & I & $\mathrm{J}$ & K & L & $\mathrm{R}(\mathrm{IK})$ & $\mathrm{T}(\mathrm{IKJ})$ & PHI & $\mathrm{T}(\mathrm{JKL})$ & $\mathrm{R}(\mathrm{KL})$ \\
\hline IC & $\mathrm{O} 1$ & $\mathrm{C} 2$ & ${ }^{*} \mathrm{C} 1$ & H1 & 1.4115 & 105.82 & -120.26 & 110.81 & 1.0905 \\
\hline & $\mathrm{O} 1$ & O5 & ${ }^{*} \mathrm{C} 1$ & $\mathrm{C} 2$ & 1.4115 & & & & 218 \\
\hline C & $\mathrm{O} 2$ & C3 & ${ }^{*} \mathrm{C} 2$ & $\mathrm{H} 2$ & 1.4190 & 110. & 16.97 & 108.77 & 1.0892 \\
\hline 10 & $\mathrm{O} 2$ & $\mathrm{C} 1$ & ${ }^{*} \mathrm{C} 2$ & $\mathrm{C} 3$ & 1.4190 & 111.74 & -123.77 & 110.04 & 1.5167 \\
\hline IC & $\mathrm{O} 3$ & $\mathrm{C} 4$ & $* \mathrm{C} 3$ & $\mathrm{H} 3$ & 1.4198 & 107.51 & 119.38 & 108.28 & 1.0957 \\
\hline 10 & $\mathrm{O} 3$ & $\mathrm{C} 2$ & $* \mathrm{C} 3$ & $\mathrm{C} 4$ & 1.4198 & 111.19 & 119.03 & 110.08 & 1.5102 \\
\hline & O4 & C5 & $* \mathrm{C} 4$ & H4 & 1.4163 & 107.94 & -120.61 & 109.07 & 1.09 \\
\hline$c$ & O4 & C3 & $* \mathrm{C} 4$ & C5 & 1.4163 & 111.43 & -118.91 & 108.83 & 1.5171 \\
\hline [C & C6 & O5 & $* \mathrm{C} 5$ & H5 & 1.5099 & 105.42 & 117.88 & 109.66 & 1.0926 \\
\hline IC & C6 & $\mathrm{C} 4$ & $* \mathrm{C} 5$ & O5 & 1.5099 & 112.84 & 117.26 & 109.77 & 1.4384 \\
\hline IC & O61 & C5 & ${ }^{*} \mathrm{C} 6$ & O62 & 1.4163 & 111.44 & 180.00 & 108.72 & 1.0943 \\
\hline$\pi$ & O5 & $\mathrm{C} 1$ & $\mathrm{C} 2$ & $\mathrm{C} 3$ & 1.4059 & 111.32 & 53.56 & 0.04 & 1.5167 \\
\hline ( & $\mathrm{C} 1$ & $\mathrm{C} 2$ & C3 & $\mathrm{C} 4$ & 1.5218 & 110.04 & -54.55 & 110.08 & 1.5102 \\
\hline [C & $\mathrm{C} 2$ & C3 & $\mathrm{C} 4$ & C5 & 1.5167 & 110.08 & 57.15 & 108.83 & 1.5171 \\
\hline IC & C3 & $\mathrm{C} 4$ & C5 & O5 & 1.5102 & 108.83 & -58.25 & 109.77 & 1.4384 \\
\hline IC & $\mathrm{C} 4$ & C5 & O5 & $\mathrm{C} 1$ & 1.5171 & 109.77 & 59.83 & 114.27 & 1.4059 \\
\hline & C5 & O5 & $\mathrm{C} 1$ & $\mathrm{C} 2$ & 1.4384 & 114.27 & -57.30 & 111.32 & 1.5218 \\
\hline [C & $\mathrm{C} 4$ & C5 & C6 & O61 & 1.5171 & 112.84 & -120.00 & 111.44 & 1.4163 \\
\hline IC & O5 & $\mathrm{C} 1$ & $\mathrm{O} 1$ & HO1 & 1.4059 & 113.00 & 67.18 & 108.04 & 0.9634 \\
\hline & $\mathrm{C} 1$ & $\mathrm{C} 2$ & $\mathrm{O} 2$ & $\mathrm{HO} 2$ & 1.5218 & 111.74 & -44.11 & 105.37 & 0.9665 \\
\hline & $\mathrm{C} 2$ & C3 & $\mathrm{O} 3$ & $\mathrm{HO} 3$ & 1.5167 & 111.19 & -51.80 & 106.26 & 0.9641 \\
\hline & C3 & $\mathrm{C} 4$ & $\mathrm{O} 4$ & $\mathrm{HO} 4$ & 1.5102 & 111.43 & 47.58 & 105.89 & 0.9645 \\
\hline
\end{tabular}

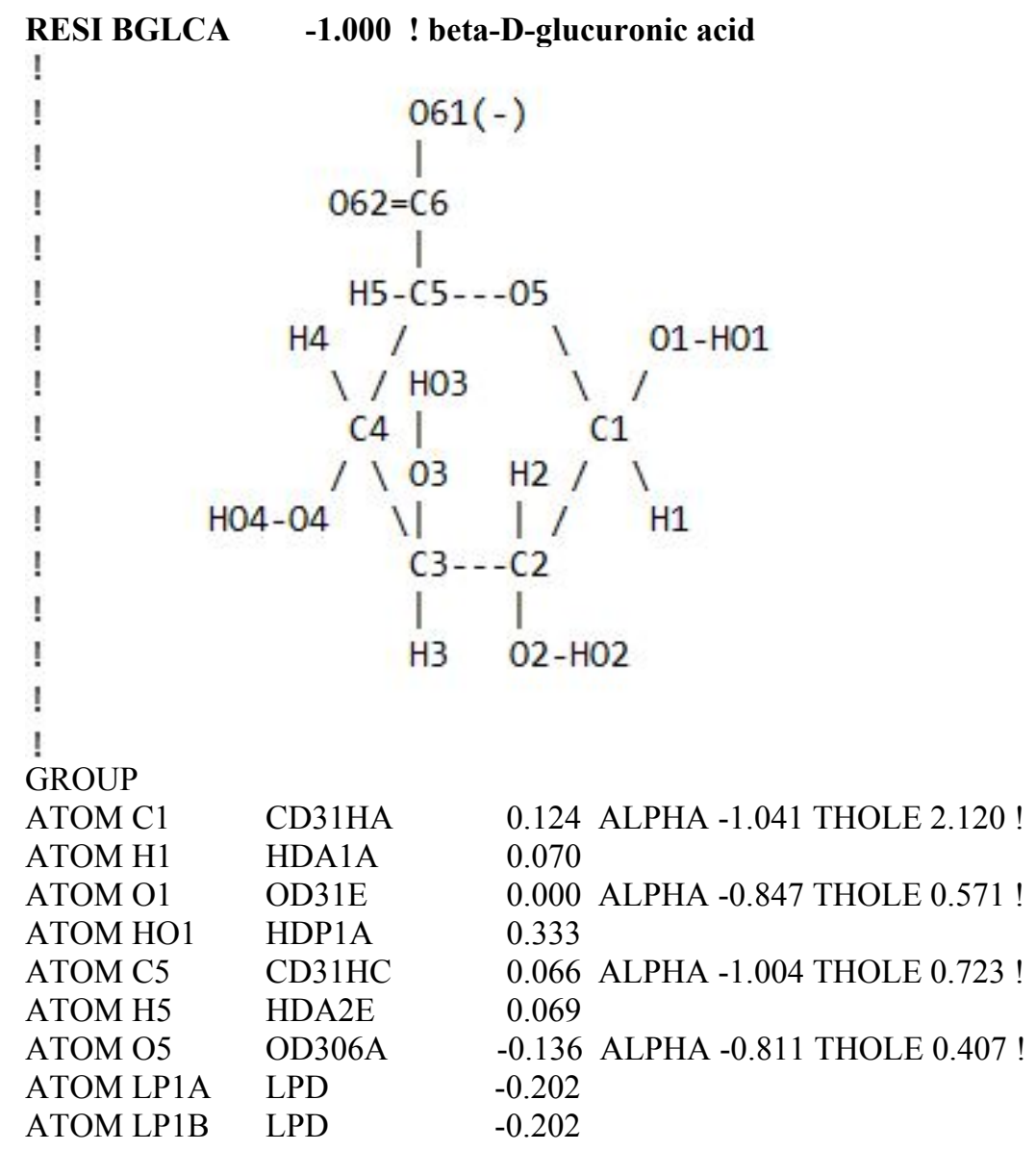




\begin{tabular}{|c|c|c|}
\hline ATOM LP5A & LPD & -0.152 \\
\hline ATOM LP5B & LPD & -0.152 \\
\hline ATOM LPX5 & LPD & 0.000 !dummy for anisotropic polarizability \\
\hline \multicolumn{3}{|l|}{ GROUP } \\
\hline ATOM C2 & CD31HB & -0.006 ALPHA -1.090 THOLE 0.208 \\
\hline ATOM H2 & HDA1A & 0.070 \\
\hline ATOM O2 & OD31E & 0.000 ALPHA -0.618 THOLE 0.213 \\
\hline ATOM HO2 & HDP1A & 0.322 \\
\hline ATOM LP2A & LPD & -0.193 \\
\hline ATOM LP2B & LPD & -0.193 \\
\hline \multicolumn{3}{|l|}{ GROUP } \\
\hline ATOM C3 & CD31HB & -0.006 ALPHA -1.007 THOLE 1.735 \\
\hline ATOM H3 & HDA1A & 0.070 \\
\hline ATOM O3 & OD31E & 0.000 ALPHA -0.502 THOLE 0.929 \\
\hline ATOM HO3 & HDP1A & 0.322 \\
\hline ATOM LP3A & LPD & -0.193 \\
\hline ATOM LP3B & LPD & -0.193 \\
\hline \multicolumn{3}{|l|}{ GROUP } \\
\hline ATOM C4 & CD31HB & -0.006 ALPHA -1.031 THOLE 0.552 \\
\hline ATOM H4 & HDA1A & 0.070 \\
\hline ATOM O4 & OD31E & 0.000 ALPHA -0.902 THOLE 2.077 \\
\hline ATOM HO4 & HDP1A & 0.322 \\
\hline ATOM LP4A & LPD & -0.193 \\
\hline ATOM LP4B & LPD & -0.193 \\
\hline \multicolumn{3}{|l|}{ GROUP } \\
\hline ATOM C6 & $\mathrm{CD} 2 \mathrm{O} 2 \mathrm{~A}$ & 0.708 ALPHA -1.016 THOLE 0.899 \\
\hline ATOM O61 & $\mathrm{OD} 2 \mathrm{C} 2 \mathrm{~A}$ & 0.003 ALPHA -0.699 THOLE 2.399 \\
\hline ATOM O62 & $\mathrm{OD} 2 \mathrm{C} 2 \mathrm{~A}$ & 0.003 ALPHA -0.699 THOLE 2.399 \\
\hline ATOM LPT1 & LPD & -0.383 \\
\hline ATOM LPT2 & LPD & -0.383 \\
\hline ATOM LPT3 & LPD & -0.383 \\
\hline ATOM LPT4 & LPD & -0.383 \\
\hline
\end{tabular}

LONEPAIR relative LP1A O1 C1 HO1 distance 0.35 angle 110.0 dihe 91.0 LONEPAIR relative LP1B O1 C1 HO1 distance 0.35 angle 110.0 dihe 269.0 ANISOTROPY O1 C1 LP1A LP1B A11 0.8108 A22 1.2162

LONEPAIR relative LP2A O2 C2 HO2 distance 0.35 angle 110.0 dihe 91.0 LONEPAIR relative LP2B O2 C2 HO2 distance 0.35 angle 110.0 dihe 269.0 ANISOTROPY O2 C2 LP2A LP2B A11 0.8108 A22 1.2162

LONEPAIR relative LP3A O3 C3 HO3 distance 0.35 angle 110.0 dihe 91.0 LONEPAIR relative LP3B O3 C3 HO3 distance 0.35 angle 110.0 dihe 269.0 ANISOTROPY O3 C3 LP3A LP3B A11 0.8108 A22 1.2162 
LONEPAIR relative LP4A O4 C4 HO4 distance 0.35 angle 110.0 dihe 91.0 LONEPAIR relative LP4B O4 C4 HO4 distance 0.35 angle 110.0 dihe 269.0 ANISOTROPY O4 C4 LP4A LP4B A11 0.8108 A22 1.2162

LONEPAIR bisector LP5A O5 C1 C5 distance 0.35 angle 110.0 dihe 90.0 LONEPAIR bisector LP5B O5 C1 C5 distance 0.35 angle 110.0 dihe 270.0 LONEPAIR bisector LPX5 O5 C1 C5 distance 0.10 angle 0.0 dihe 0.0 ANISOTROPY O5 LPX5 LP5A LP5B A11 0.8889 A22 1.2222

LONEPAIR relative LPT1 O61 C6 O62 distance 0.35 angle 110.0 dihe 0.0 LONEPAIR relative LPT2 O61 C6 O62 distance 0.35 angle 110.0 dihe 180.0 LONEPAIR relative LPT3 O62 C6 O61 distance 0.35 angle 110.0 dihe 0.0 LONEPAIR relative LPT4 O62 C6 O61 distance 0.35 angle 110.0 dihe 180.0 ANISOTROPY O61 C6 LPT1 LPT2 A11 0.7229 A22 1.265 ANISOTROPY O62 C6 LPT3 LPT4 A11 0.7229 A22 1.265

\begin{tabular}{|c|c|c|c|c|c|c|c|c|c|}
\hline & 1 & $\mathrm{~J}$ & K & L & $\mathrm{R}(\mathrm{IK})$ & $\mathrm{T}(\mathrm{IKJ})$ & PHI & $\mathrm{T}(\mathrm{JKI}$ & $\mathrm{R}(\mathrm{K}$ \\
\hline IC & $\mathrm{O} 1$ & $\mathrm{C} 2$ & ${ }^{*} \mathrm{C} 1$ & $\mathrm{H} 1$ & 1.4115 & 105.82 & 120.26 & 110.81 & 1.0905 \\
\hline [C & $\mathrm{O} 1$ & O5 & ${ }^{*} \mathrm{C} 1$ & $\mathrm{C} 2$ & 1.4115 & 113.00 & 118.92 & 111.32 & 1.5218 \\
\hline C & $\mathrm{O} 2$ & $\mathrm{C} 3$ & ${ }^{*} \mathrm{C} 2$ & $\mathrm{H} 2$ & 1.4190 & 110.99 & -116.97 & 108.77 & 1.0892 \\
\hline & $\mathrm{O} 2$ & $\mathrm{C} 1$ & ${ }^{*} \mathrm{C} 2$ & $\mathrm{C} 3$ & 1.4190 & 111.7 & -123.77 & 110.04 & 1.01 \\
\hline & $\mathrm{O} 3$ & $\mathrm{C} 4$ & $* \mathrm{C} 3$ & $\mathrm{H} 3$ & 1.4198 & 107.51 & 119.38 & 108.28 & 10957 \\
\hline [C & $\mathrm{O} 3$ & $\mathrm{C} 2$ & $* \mathrm{C} 3$ & $\mathrm{C} 4$ & 1.4198 & 111.19 & 119.03 & 110.08 & 1.5102 \\
\hline [C & O4 & $\mathrm{C} 5$ & $* \mathrm{C} 4$ & $\mathrm{H} 4$ & 1.4163 & 107.94 & -120.61 & 109.07 & 1.0972 \\
\hline IC & O4 & $\mathrm{C} 3$ & $* \mathrm{C} 4$ & $\mathrm{C} 5$ & 1.4163 & 111.43 & -118.91 & 108.83 & 1.5171 \\
\hline 10 & C6 & O5 & $* \mathrm{C} 5$ & H5 & 1.5099 & 105.42 & 120.00 & 109.66 & 1.0926 \\
\hline IC & C6 & $\mathrm{C} 4$ & ${ }^{*} \mathrm{C} 5$ & O5 & 1.5099 & 112.84 & 120.00 & 109.77 & 1.4384 \\
\hline [C & O61 & C5 & ${ }^{*} \mathrm{C} 6$ & O62 & 1.4163 & 111.44 & 180.00 & 108.72 & 1.0943 \\
\hline [C & O5 & $\mathrm{C} 1$ & $\mathrm{C} 2$ & $\mathrm{C} 3$ & 1.4059 & 111.32 & 53.56 & 110.04 & 1.5167 \\
\hline IC & $\mathrm{C} 1$ & $\mathrm{C} 2$ & $\mathrm{C} 3$ & $\mathrm{C} 4$ & 1.5218 & 110.04 & -54.55 & 110.08 & 1.5102 \\
\hline IC & $\mathrm{C} 2$ & C3 & $\mathrm{C} 4$ & C5 & 1.5167 & 110.08 & 57.15 & 108.83 & 1.5171 \\
\hline & C3 & $\mathrm{C} 4$ & C5 & O5 & 1.5102 & 108.83 & -58.25 & 109.77 & 1.4384 \\
\hline [C & $\mathrm{C} 4$ & $\mathrm{C} 5$ & O5 & $\mathrm{C} 1$ & 1.5171 & 109.77 & 59.83 & 114.27 & 1.4059 \\
\hline [C & $\mathrm{C} 5$ & O5 & $\mathrm{C} 1$ & $\mathrm{C} 2$ & 1.4384 & 114.27 & -57.30 & 111.32 & 1.5218 \\
\hline [C & $\mathrm{C} 4$ & C5 & C6 & O61 & 1.5171 & 112.84 & -120.00 & 111.44 & 1.4163 \\
\hline & O5 & $\mathrm{C} 1$ & $\mathrm{O} 1$ & HO1 & 1.4059 & 113.00 & 67.18 & 108.04 & 0.9634 \\
\hline & $\mathrm{C} 1$ & $\mathrm{C} 2$ & $\mathrm{O} 2$ & $\mathrm{HO} 2$ & 1.5218 & 111.74 & -44.11 & 105.37 & 0.9665 \\
\hline & $\mathrm{C} 2$ & C3 & $\mathrm{O} 3$ & HO3 & 1.5167 & 111.19 & -51.80 & 106.26 & 0.9641 \\
\hline & $\mathrm{C} 3$ & $\mathrm{C} 4$ & O4 & HO4 & .5102 & 111.43 & 47.58 & 105.89 & 0.9645 \\
\hline
\end{tabular}

RESI AIDOA $\quad-1.000$ ! alpha-L-iduronic acid 


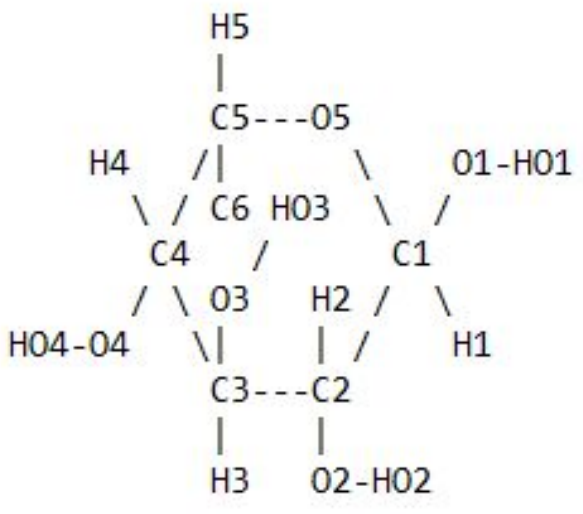

n.b.: 061 and 062 are attached to $C 6$<smiles>CCOC(=O)O[Na]</smiles>

GROUP

ATOM C1

CD31HA $\quad 0.124$ ALPHA -1.041 THOLE 2.120!

ATOM H1

HDA1A

0.070

OD31E $\quad 0.000$ ALPHA -0.847 THOLE 0.571!

ATOM HO1

HDP1A

0.333

ATOM C5

CD31HC

0.066 ALPHA -1.004 THOLE 0.723 !

ATOM H5

HDA2E

0.069

ATOM O5

OD306A

ATOM LP1A

LPD

-0.136 ALPHA -0.811 THOLE 0.407 !

ATOM LP1B

LPD

$-0.202$

$-0.202$

ATOM LP5A

LPD

LPD

ATOM LPX5 LPD

GROUP

ATOM C2

CD31HB

HDA1A

$-0.152$

$-0.152$

0.000 !dummy for anisotropic polarizability

ATOM H2

ATOM O2

OD31E

ATOM HO2

HDP1A

LPD

ATOM LP2A

ATOM LP2B

LPD

GROUP

ATOM C3

CD31HB

-0.006 ALPHA -1.090 THOLE 0.208

0.070

0.000 ALPHA -0.618 THOLE 0.213

0.322

$-0.193$

$-0.193$

ATOM H3

HDA1A

-0.006 ALPHA -1.007 THOLE 1.735

ATOM O3

OD31E

ATOM HO3

HDP1A

0.070

0.000 ALPHA -0.502 THOLE 0.929

0.322

$-0.193$

ATOM LP3B

GROUP

ATOM C4

LPD

$-0.193$

ATOM H4

LPD

CD31HB

-0.006 ALPHA -1.031 THOLE 0.552

HDA1A

0.070

OD31E

ATOM HO4

HDP1A

0.000 ALPHA -0.902 THOLE 2.077

ATOM LP4A

LPD

0.322

$-0.193$

ATOM LP4B

LPD

$-0.193$

GROUP

ATOM C6

CD2O2A

0.708 ALPHA -1.016 THOLE 0.899

ATOM O61

OD2C2A

0.003 ALPHA -0.699 THOLE 2.399 


\begin{tabular}{|c|c|c|}
\hline ATOM O62 & $\mathrm{OD} 2 \mathrm{C} 2 \mathrm{~A}$ & 0.003 ALPHA -0.699 THOLE 2.399 \\
\hline ATOM LPT1 & LPD & -0.383 \\
\hline ATOM LPT2 & LPD & -0.383 \\
\hline ATOM LPT3 & LPD & -0.383 \\
\hline ATOM LPT4 & LPD & -0.383 \\
\hline
\end{tabular}

LONEPAIR relative LP1A O1 C1 HO1 distance 0.35 angle 110.0 dihe 91.0 LONEPAIR relative LP1B O1 C1 HO1 distance 0.35 angle 110.0 dihe 269.0 ANISOTROPY O1 C1 LP1A LP1B A11 0.8108 A22 1.2162

LONEPAIR relative LP2A O2 $\mathrm{C} 2 \mathrm{HO} 2$ distance 0.35 angle 110.0 dihe 91.0 LONEPAIR relative LP2B O2 C2 HO2 distance 0.35 angle 110.0 dihe 269.0 ANISOTROPY O2 C2 LP2A LP2B A11 0.8108 A22 1.2162

LONEPAIR relative LP3A O3 C3 HO3 distance 0.35 angle 110.0 dihe 91.0 LONEPAIR relative LP3B O3 C3 HO3 distance 0.35 angle 110.0 dihe 269.0 ANISOTROPY O3 C3 LP3A LP3B A11 0.8108 A22 1.2162

LONEPAIR relative LP4A O4 C4 HO4 distance 0.35 angle 110.0 dihe 91.0 LONEPAIR relative LP4B O4 C4 HO4 distance 0.35 angle 110.0 dihe 269.0 ANISOTROPY O4 C4 LP4A LP4B A11 0.8108 A22 1.2162

LONEPAIR bisector LP5A O5 C1 C5 distance 0.35 angle 110.0 dihe 90.0 LONEPAIR bisector LP5B O5 C1 C5 distance 0.35 angle 110.0 dihe 270.0 LONEPAIR bisector LPX5 O5 C1 C5 distance 0.10 angle 0.0 dihe 0.0 ANISOTROPY O5 LPX5 LP5A LP5B A11 0.8889 A22 1.2222

LONEPAIR relative LPT1 O61 C6 O62 distance 0.35 angle 110.0 dihe 0.0 LONEPAIR relative LPT2 O61 C6 O62 distance 0.35 angle 110.0 dihe 180.0 LONEPAIR relative LPT3 O62 C6 O61 distance 0.35 angle 110.0 dihe 0.0 LONEPAIR relative LPT4 O62 C6 O61 distance 0.35 angle 110.0 dihe 180.0 ANISOTROPY O61 C6 LPT1 LPT2 A11 0.7229 A22 1.265 ANISOTROPY O62 C6 LPT3 LPT4 A11 0.7229 A22 1.265

$\begin{array}{lccccccccc}\text { ! } & \mathrm{I} & \mathrm{J} & \mathrm{K} & \mathrm{L} & \mathrm{R}(\mathrm{IK}) & \mathrm{T}(\mathrm{IKJ}) & \mathrm{PHI} & \mathrm{T}(\mathrm{JKL}) & \mathrm{R}(\mathrm{KL}) \\ \mathrm{IC} & \mathrm{O} 1 & \mathrm{C} 2 & * \mathrm{C} 1 & \mathrm{H} 1 & 1.4115 & 105.82 & 120.26 & 110.81 & 1.0905 \\ \mathrm{IC} & \mathrm{O} 1 & \mathrm{O} 5 & * \mathrm{C} 1 & \mathrm{C} 2 & 1.4115 & 113.00 & 118.92 & 111.32 & 1.5218 \\ \mathrm{IC} & \text { O2 } & \mathrm{C} 3 & * \mathrm{C} 2 & \mathrm{H} 2 & 1.4190 & 108.02 & -120.00 & 108.77 & 1.0892 \\ \mathrm{IC} & \mathrm{O} 2 & \mathrm{C} 1 & * \mathrm{C} 2 & \mathrm{C} 3 & 1.4190 & 111.74 & -120.00 & 110.04 & 1.5167 \\ \mathrm{IC} & \mathrm{O} 3 & \mathrm{C} 4 & * \mathrm{C} 3 & \mathrm{H} 3 & 1.4198 & 108.29 & 120.00 & 108.28 & 1.0957 \\ \mathrm{IC} & \text { O3 } & \mathrm{C} 2 & * \mathrm{C} 3 & \mathrm{C} 4 & 1.4198 & 111.19 & 120.00 & 110.08 & 1.5102 \\ \mathrm{IC} & \text { O4 } & \mathrm{C} 5 & * \mathrm{C} 4 & \mathrm{H} 4 & 1.4163 & 108.82 & -120.00 & 109.07 & 1.0972 \\ \mathrm{IC} & \text { O4 } & \mathrm{C} 3 & * \mathrm{C} 4 & \mathrm{C} 5 & 1.4163 & 111.43 & -120.00 & 108.83 & 1.5171 \\ \mathrm{IC} & \mathrm{C} 6 & \mathrm{O} 5 & * \mathrm{C} 5 & \mathrm{H} 5 & 1.5099 & 105.42 & -117.88 & 109.66 & 1.0926 \\ \mathrm{IC} & \mathrm{C} 6 & \mathrm{C} 4 & * \mathrm{C} 5 & \text { O5 } & 1.5099 & 112.84 & -117.26 & 109.77 & 1.4384\end{array}$


$\begin{array}{lllllllll}\text { IC } & \text { O61 C5 *C6 O62 } & 1.4163 & 111.44 & 180.00 & 108.72 & 1.0943\end{array}$

$\begin{array}{llllllllll}\text { IC } & \text { O5 } & \text { C1 } & \text { C2 } & \text { C3 } & 1.4059 & 111.32 & -53.56 & 110.04 & 1.5167\end{array}$

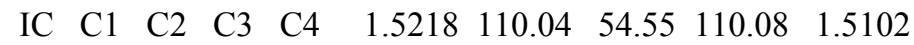

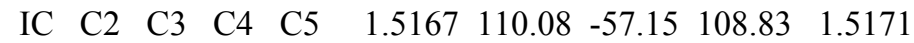

$\begin{array}{llllllllll}\text { IC } & \mathrm{C} 3 & \mathrm{C} 4 & \mathrm{C} 5 & \mathrm{O} 5 & 1.5102 & 108.83 & 58.25 & 109.77 & 1.4384\end{array}$

$\begin{array}{lllllllllll}\text { IC } & \mathrm{C} 4 & \mathrm{C} 5 & \mathrm{O} 5 & \mathrm{C} 1 & 1.5171 & 109.77 & -59.84 & 114.27 & 1.4059\end{array}$

$\begin{array}{llllllllll}\text { IC } & \mathrm{C} 5 & \mathrm{O} 5 & \mathrm{C} 1 & \mathrm{C} 2 & 1.4384 & 114.27 & 57.30 & 111.32 & 1.5218\end{array}$

$\begin{array}{lllllllllll}\text { IC } & \text { C4 } & \text { C5 } & \text { C6 } & \text { O661 } & 1.5171 & 112.84 & 120.00 & 111.44 & 1.4163\end{array}$

$\begin{array}{llllllllll}\text { IC } & \text { O5 } & \text { C1 } & \text { O1 } & \text { HO1 } & 1.4059 & 113.00 & -60.00 & 108.04 & 0.9634\end{array}$

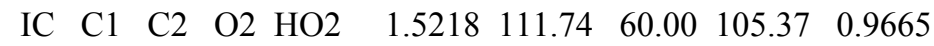

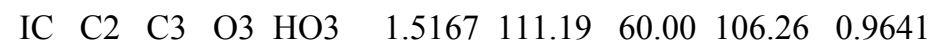

$\begin{array}{llllllllll}\text { IC } & \mathrm{C} 3 & \mathrm{C} 4 & \mathrm{O} 4 & \mathrm{HO} & 1.5102 & 111.43 & -60.00 & 105.89 & 0.9645\end{array}$

PATCH FIRST NONE LAST NONE

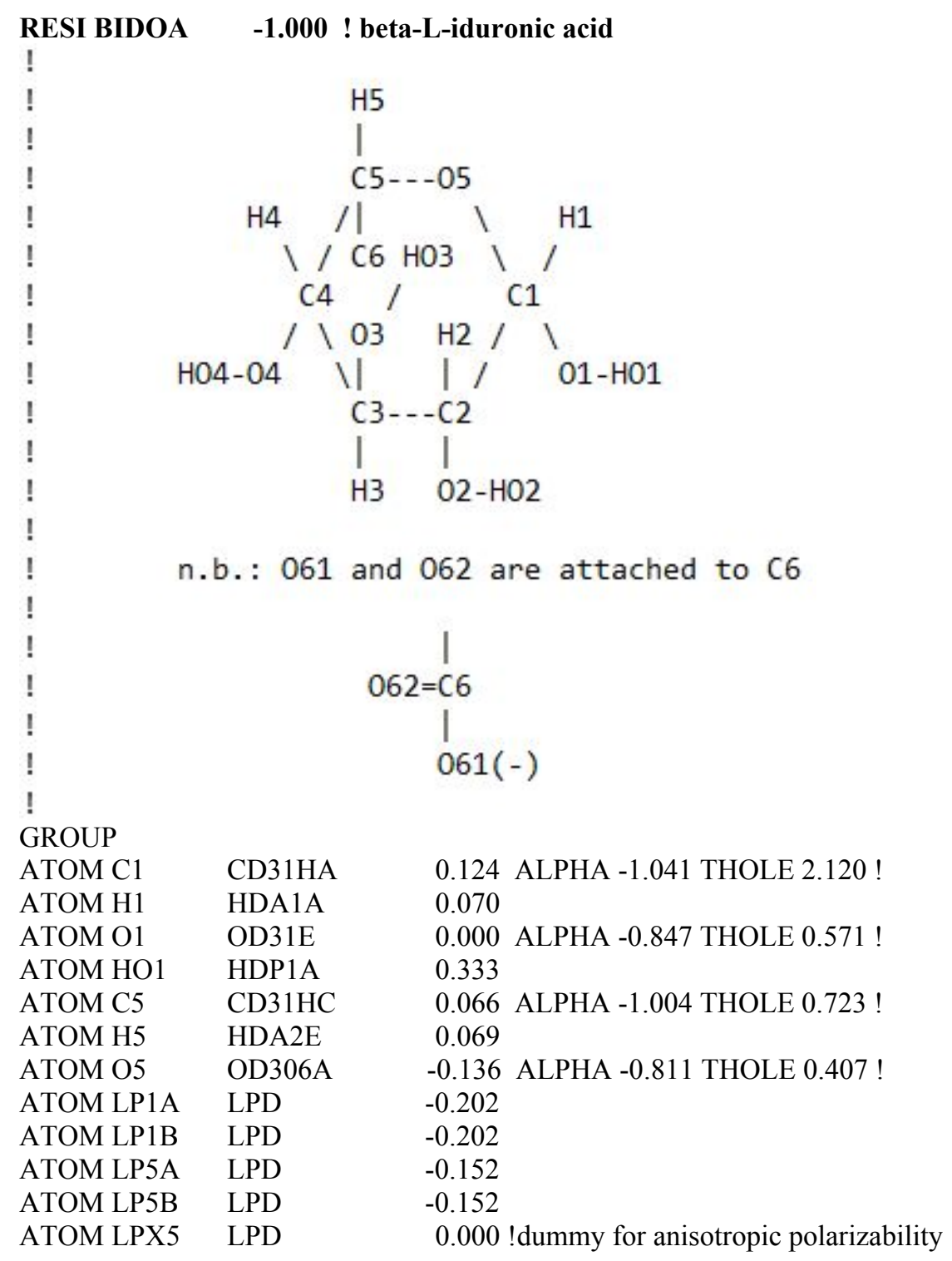




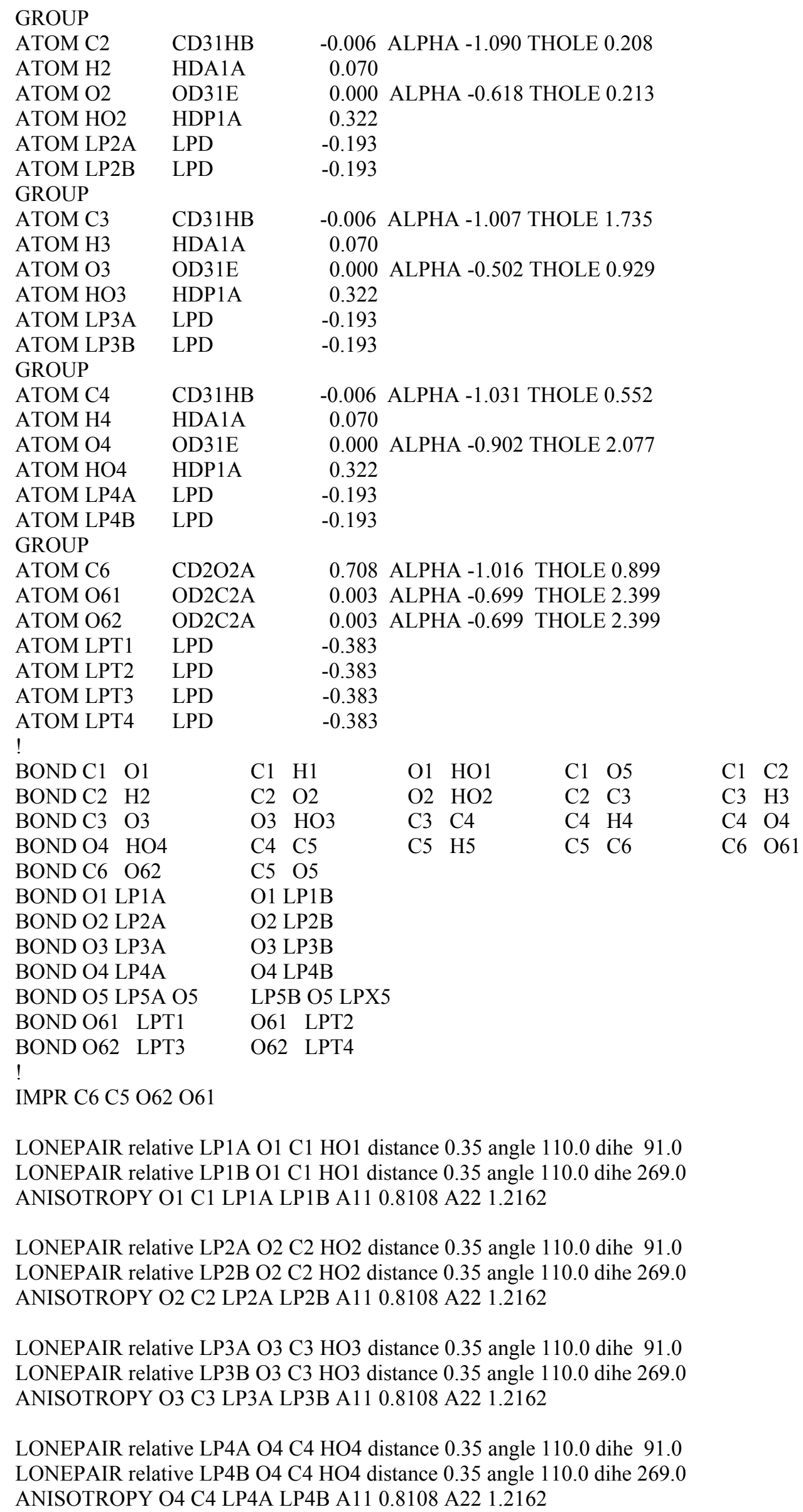


LONEPAIR bisector LP5A O5 C1 C5 distance 0.35 angle 110.0 dihe 90.0 LONEPAIR bisector LP5B O5 C1 C5 distance 0.35 angle 110.0 dihe 270.0 LONEPAIR bisector LPX5 O5 C1 C5 distance 0.10 angle 0.0 dihe 0.0 ANISOTROPY O5 LPX5 LP5A LP5B A11 0.8889 A22 1.2222

LONEPAIR relative LPT1 O61 C6 O62 distance 0.35 angle 110.0 dihe 0.0 LONEPAIR relative LPT2 O61 C6 O62 distance 0.35 angle 110.0 dihe 180.0 LONEPAIR relative LPT3 O62 C6 O61 distance 0.35 angle 110.0 dihe 0.0 LONEPAIR relative LPT4 O62 C6 O61 distance 0.35 angle 110.0 dihe 180.0 ANISOTROPY O61 C6 LPT1 LPT2 A11 0.7229 A22 1.265

ANISOTROPY O62 C6 LPT3 LPT4 A11 0.7229 A22 1.265

\begin{tabular}{|c|c|c|c|c|c|c|c|c|c|}
\hline | & I & $\mathrm{J}$ & K & $\mathrm{L}$ & $\mathrm{R}(\mathrm{IK})$ & $\mathrm{T}(\mathrm{IKJ})$ & PHI & $\mathrm{T}(\mathrm{JKL})$ & $\mathrm{R}(\mathrm{KL})$ \\
\hline IC & $\mathrm{O} 1$ & $\mathrm{C} 2$ & ${ }^{*} \mathrm{C} 1$ & $\mathrm{H} 1$ & 1.4115 & 105.82 & -120.26 & 110.81 & 1.0905 \\
\hline $\mathrm{C}$ & $\mathrm{O} 1$ & $\mathrm{O} 5$ & ${ }^{*} \mathrm{C} 1$ & $\mathrm{C} 2$ & 1.4115 & 113.0 & 8.92 & 111.32 & 1.5218 \\
\hline & $\mathrm{O} 2$ & C3 & $* \mathrm{C} 2$ & $\mathrm{H} 2$ & 1.4190 & 108.0 & 20.00 & 108.77 & 1.0892 \\
\hline & $\mathrm{O} 2$ & $\mathrm{C} 1$ & $* \mathrm{C} 2$ & $\mathrm{C} 3$ & 1.4190 & 111.7 & -120.00 & 110.04 & 1.5167 \\
\hline & $\mathrm{O} 3$ & $\mathrm{C} 4$ & $* \mathrm{C} 3$ & H3 & 1.4198 & 108.29 & 120.00 & 108.28 & 1.0957 \\
\hline & $\mathrm{O} 3$ & $\mathrm{C} 2$ & ${ }^{*} \mathrm{C} 3$ & $\mathrm{C} 4$ & & 11 & 120.00 & 08 & 1.5102 \\
\hline & $\mathrm{O} 4$ & $\mathrm{C} 5$ & $* \mathrm{C} 4$ & $\mathrm{H} 4$ & 1.4163 & 108.82 & -120.00 & 109.07 & 1.0972 \\
\hline & $\mathrm{O} 4$ & $\mathrm{C} 3$ & $* \mathrm{C} 4$ & $\mathrm{C} 5$ & 1.4163 & 111.4 & 20.00 & 108.83 & 1.5171 \\
\hline & C6 & O5 & $* \mathrm{C} 5$ & H5 & 1.5099 & 10 & 88 & 109.66 & 1.0926 \\
\hline & C6 & $\mathrm{C} 4$ & $* \mathrm{C} 5$ & O5 & 1.5099 & 112 & -117.26 & 109.77 & 1.4384 \\
\hline & O61 & $\mathrm{C} 5$ & *C6 & O62 & 1.4 & 44 & 180.00 & 108.72 & 1.0943 \\
\hline IC & $\mathrm{O} 5$ & $\mathrm{C} 1$ & $\mathrm{C} 2$ & $\mathrm{C} 3$ & 1.4059 & 111.32 & -53.56 & 110.04 & 1.5167 \\
\hline & $\mathrm{C} 1$ & $\mathrm{C} 2$ & $\mathrm{C} 3$ & $\mathrm{C} 4$ & 1.5218 & 110.04 & 54.55 & 110.08 & 1.5102 \\
\hline & $\mathrm{C} 2$ & $\mathrm{C} 3$ & $\mathrm{C} 4$ & $\mathrm{C} 5$ & 1.5167 & 110.08 & -57.15 & 108.83 & 1.5171 \\
\hline & $\mathrm{C} 3$ & $\mathrm{C} 4$ & $\mathrm{C} 5$ & O5 & 1.5102 & 108.83 & 58.25 & 109.77 & 1.4384 \\
\hline & $\mathrm{C} 4$ & C5 & O5 & $\mathrm{C} 1$ & 1.5171 & 109.77 & -59.84 & 114.27 & 1.4059 \\
\hline & $\mathrm{C} 5$ & O5 & $\mathrm{C} 1$ & $\mathrm{C} 2$ & 1.4384 & 114.27 & 57.30 & 111.32 & 1.5218 \\
\hline & $\mathrm{C} 4$ & $\mathrm{C} 5$ & C6 & O61 & 1.5171 & 112.84 & 120.00 & 111.44 & 1.4163 \\
\hline & $\mathrm{O} 5$ & $\mathrm{C} 1$ & $\mathrm{O} 1$ & HO1 & 1.4059 & 113.00 & -60.00 & 108.04 & 0.9634 \\
\hline & $\mathrm{C} 1$ & $\mathrm{C} 2$ & $\mathrm{O} 2$ & $\mathrm{HO} 2$ & 1.5218 & 111.74 & 60.00 & 105.37 & 0.9665 \\
\hline & $\mathrm{C} 2$ & C3 & $\mathrm{O} 3$ & HO3 & 1.5167 & 111.19 & 60.00 & 106.26 & 0.9641 \\
\hline & $\mathrm{C} 3$ & $\mathrm{C} 4$ & $\mathrm{O} 4$ & $\mathrm{HO} 4$ & 1.5102 & 111.43 & -60.00 & 105.89 & 0.9645 \\
\hline
\end{tabular}

PATCH FIRST NONE LAST NONE

RESI AGLCNA $\quad 0.000 ! 2$-acetyl-2-deoxy-alpha-D-glucosamine ! (alpha N-acetylglucosamine or GlcNAc) 


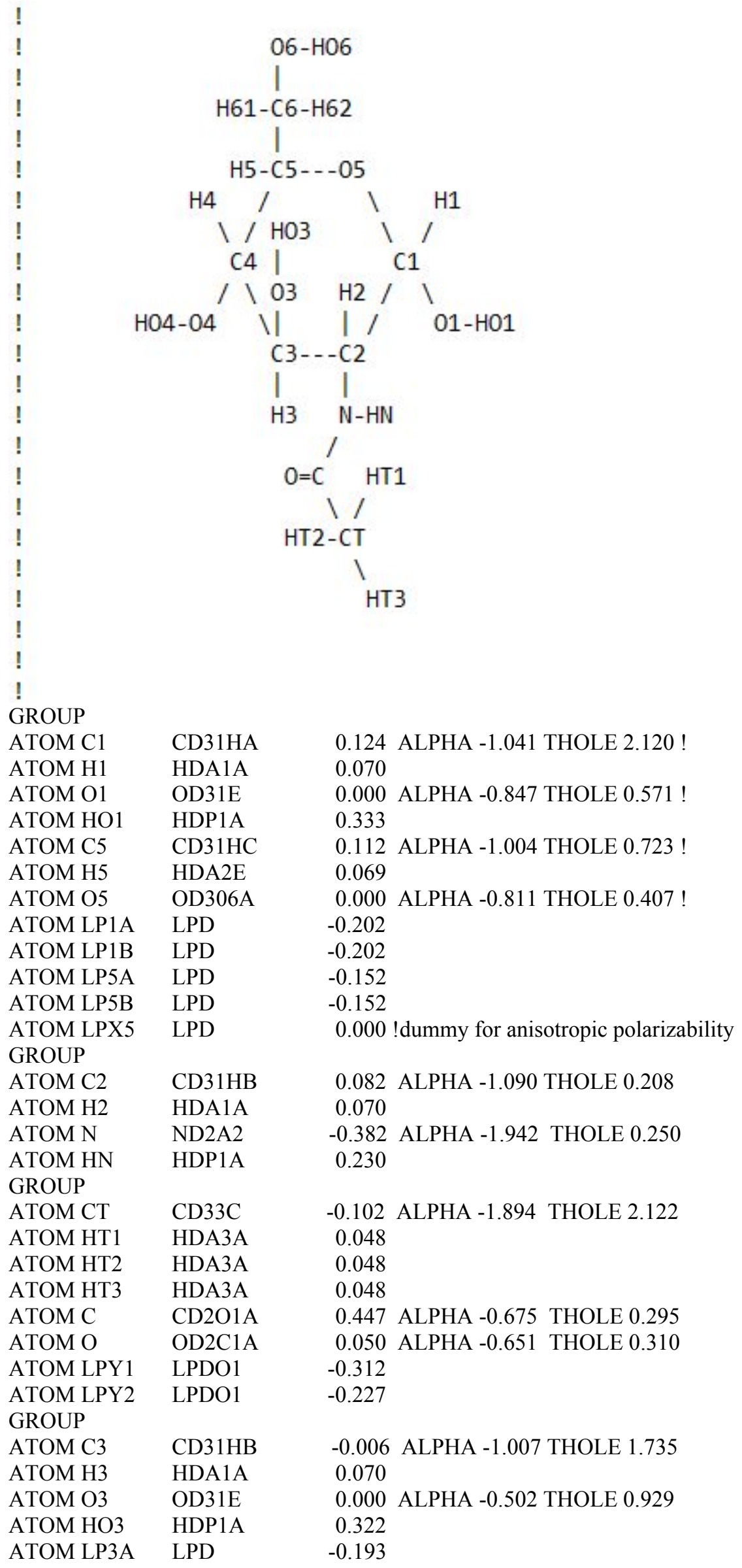




$\begin{array}{llr}\text { ATOM LP3B } & \text { LPD } & -0.193 \\ \text { GROUP } & & -0.006 \text { ALPHA }-1.031 \text { THOLE } 0.552 \\ \text { ATOM C4 } & \text { CD31HB } & 0.070 \\ \text { ATOM H4 } & \text { HDA1A } & 0.000 \text { ALPHA -0.902 THOLE } 2.077 \\ \text { ATOM O4 } & \text { OD31E } & 0.322 \\ \text { ATOM HO4 } & \text { HDP1A } & -0.193 \\ \text { ATOM LP4A } & \text { LPD } & -0.193 \\ \text { ATOM LP4B } & \text { LPD } & \\ \text { GROUP } & & -0.086 \text { ALPHA -1.005 THOLE } 0.249 ! \\ \text { ATOM C6 } & \text { CD32A } & 0.080 \\ \text { ATOM H61 } & \text { HDA2A } & 0.080 \\ \text { ATOM H62 } & \text { HDA2A } & 0.000 \text { ALPHA -0.778 THOLE } 0.207 \text { ! } \\ \text { ATOM O6 } & \text { OD31A } & 0.328 \\ \text { ATOM HO6 } & \text { HDP1A } & -0.201 \\ \text { ATOM LP6A } & \text { LPD } & -0.201\end{array}$

!

BOND C1 O1

BOND C2 H2

BOND C3 O3

BOND O4 HO4

\section{C1 H1}

$\mathrm{C} 2 \mathrm{~N}$

O1 HO1

C1 O5

N HN

C2 $\mathrm{C} 3$

$\mathrm{O} 3 \mathrm{HO} 3$

C3 C4

$\mathrm{C} 5 \mathrm{H} 5$

$\mathrm{C} 4 \mathrm{H} 4$

C4 C5

O6 HO6

C5 C6

C6 O6

BOND C $\mathrm{O}$

C CT

O1 LP1B

CT HT1

C5 O5

BOND O1 LP1A

O3 LP3B

BOND O3 LP3A

O4 LP4B

BOND O4 LP4A

O5 LP5B

O6 LP6B

BOND O6 LP6A

O LPY2

\section{O5 LPX5}

BOND O LPY1

IMPR C CT N O

IMPR N C C2 HN

CT HT2

C1 C2

C3 H3

C4 O4

C6 H61

$\mathrm{N}$ C

CT HT3

LONEPAIR relative LP1A O1 C1 HO1 distance 0.35 angle 110.0 dihe 91.0 LONEPAIR relative LP1B O1 C1 HO1 distance 0.35 angle 110.0 dihe 269.0 ANISOTROPY O1 C1 LP1A LP1B A11 0.8108 A22 1.2162

LONEPAIR relative LP3A O3 C3 HO3 distance 0.35 angle 110.0 dihe 91.0 LONEPAIR relative LP3B O3 C3 HO3 distance 0.35 angle 110.0 dihe 269.0 ANISOTROPY O3 C3 LP3A LP3B A11 0.8108 A22 1.2162

LONEPAIR relative LP4A O4 C4 HO4 distance 0.35 angle 110.0 dihe 91.0 LONEPAIR relative LP4B O4 C4 HO4 distance 0.35 angle 110.0 dihe 269.0 ANISOTROPY O4 C4 LP4A LP4B A11 0.8108 A22 1.2162

LONEPAIR bisector LP5A O5 C1 C5 distance 0.35 angle 110.0 dihe 90.0 LONEPAIR bisector LP5B O5 C1 C5 distance 0.35 angle 110.0 dihe 270.0 LONEPAIR bisector LPX5 O5 C1 C5 distance 0.10 angle 0.0 dihe 0.0 ANISOTROPY O5 LPX5 LP5A LP5B A11 0.8889 A22 1.2222

LONEPAIR relative LP6A O6 C6 HO6 distance 0.35 angle 110.0 dihe 91.0 LONEPAIR relative LP6B O6 C6 HO6 distance 0.35 angle 110.0 dihe 269.0 ANISOTROPY O6 C6 LP6A LP6B A11 0.8108 A22 1.2162

LONEPAIR relative LPY1 O C CT distance 0.30 angle 91.0 dihe 0.0 LONEPAIR relative LPY2 O C CT distance 0.30 angle 91.0 dihe 180.0 ANISOTROPY O C LPY1 LPY2 A11 0.82322 A22 1.14332
$\begin{array}{llllllllll}\text { ! } & \mathrm{I} & \mathrm{J} & \mathrm{K} & \mathrm{L} & \mathrm{R}(\mathrm{IK}) & \mathrm{T}(\mathrm{IKJ}) & \mathrm{PHI} & \mathrm{T}(\mathrm{JKL}) & \mathrm{R}(\mathrm{KL})\end{array}$
$\begin{array}{llllllllll}\mathrm{IC} & \mathrm{O} 1 & \mathrm{C} 2 & * \mathrm{C} 1 & \mathrm{H} 1 & 1.4115 & 105.82 & -120.26 & 110.81 & 1.0905\end{array}$
$\begin{array}{llllllllll}\mathrm{IC} & \mathrm{O} 1 & \mathrm{O} 5 & * \mathrm{C} 1 & \mathrm{C} 2 & 1.4115 & 113.00 & -118.92 & 111.32 & 1.5218\end{array}$
$\begin{array}{llllllllll}\text { IC } & \mathrm{N} & \mathrm{C} 3 & * \mathrm{C} 2 & \mathrm{H} 2 & 1.4190 & 110.99 & -116.97 & 108.77 & 1.0892\end{array}$ 


\begin{tabular}{|c|c|c|c|c|c|c|c|c|c|}
\hline C & $\mathrm{N}$ & $\mathrm{C} 1$ & ${ }^{*} \mathrm{C} 2$ & $\mathrm{C} 3$ & 90 & 11178 & 23.77 & 04 & $0 /$ \\
\hline IC & $\mathrm{O} 3$ & $\mathrm{C} 4$ & ${ }^{*} \mathrm{C} 3$ & $\mathrm{H} 3$ & 1.4198 & 107.51 & 119.38 & 108.28 & 1.0957 \\
\hline IC & $\mathrm{O} 3$ & $\mathrm{C} 2$ & ${ }^{*} \mathrm{C} 3$ & $\mathrm{C} 4$ & 1.4198 & 111.19 & 119.03 & 110.08 & 1.5102 \\
\hline IC & $\mathrm{O} 4$ & $\mathrm{C} 5$ & ${ }^{*} \mathrm{C} 4$ & $\mathrm{H} 4$ & 1.4163 & 107.94 & -120.61 & 109.07 & 1.0972 \\
\hline IC & $\mathrm{O} 4$ & $\mathrm{C} 3$ & ${ }^{*} \mathrm{C} 4$ & $\mathrm{C} 5$ & 1.4163 & 111.43 & -118.91 & 108.83 & 1.5171 \\
\hline IC & C6 & O5 & ${ }^{*} \mathrm{C} 5$ & H5 & 1.5099 & 105.42 & 117.88 & 66 & \\
\hline IC & C6 & $\mathrm{C} 4$ & ${ }^{*} \mathrm{C} 5$ & O5 & 1.5099 & 112.84 & 117.26 & 109.77 & 1.4384 \\
\hline IC & O6 & H62 & $2 * \mathrm{C}$ & H61 & 1.4163 & 111.41 & -118.10 & 108.62 & 1.0873 \\
\hline IC & O6 & $\mathrm{C} 5$ & ${ }^{*} \mathrm{C} 6$ & H62 & 1.4163 & 111.44 & -123.18 & 108.72 & 1.0943 \\
\hline IC & O5 & $\mathrm{C} 1$ & $\mathrm{C} 2$ & $\mathrm{C} 3$ & 1.4059 & 111.32 & 53.56 & 110.04 & $1.516^{\circ}$ \\
\hline IC & $\mathrm{C} 1$ & $\mathrm{C} 2$ & $\mathrm{C} 3$ & $\mathrm{C} 4$ & 1.5218 & 110.04 & -54.55 & 110.08 & 1.5102 \\
\hline IC & $\mathrm{C} 2$ & $\mathrm{C} 3$ & $\mathrm{C} 4$ & C5 & 1.5167 & 110.08 & 57.15 & 108.83 & 1.5171 \\
\hline IC & C3 & $\mathrm{C} 4$ & $\mathrm{C} 5$ & O5 & 1.5102 & 108.83 & -58.25 & 109.77 & 1.4384 \\
\hline IC & $\mathrm{C} 4$ & $\mathrm{C} 5$ & O5 & $\mathrm{C} 1$ & 1.5171 & 109.77 & 59.83 & 114.27 & 1.4059 \\
\hline IC & C5 & O5 & $\mathrm{C} 1$ & $\mathrm{C} 2$ & 1.4384 & 114.27 & -57.30 & 111.32 & 1.5218 \\
\hline IC & $\mathrm{C} 4$ & C5 & C6 & O6 & 1.5171 & 112.84 & -179.74 & 111.44 & 1.4163 \\
\hline IC & O5 & $\mathrm{C} 1$ & $\mathrm{O} 1$ & HO1 & 1.4059 & 113.00 & 67.18 & 108.04 & 0.963 \\
\hline IC & $\mathrm{C} 1$ & $\mathrm{C} 2$ & $\mathrm{~N}$ & $\mathrm{HN}$ & 1.5218 & 111.74 & -44.11 & 105.37 & 0.9665 \\
\hline IC & $\mathrm{C} 2$ & $\mathrm{C} 3$ & $\mathrm{O} 3$ & HO3 & 1.5167 & 111.19 & -51.80 & 106.26 & 0.9641 \\
\hline IC & C3 & $\mathrm{C} 4$ & $\mathrm{O} 4$ & HO4 & 1.5 & 111 & 47.58 & 105.89 & 0.9645 \\
\hline IC & $\mathrm{C} 5$ & C6 & O6 & HO6 & 1.5099 & 111.44 & -54.79 & 105.51 & 0.9630 \\
\hline IC & $\mathrm{C}$ & $\mathrm{N}$ & $\mathrm{C} 2$ & C3 & 1.0000 & 120.00 & -60.00 & 120.00 & 1.0000 \\
\hline IC & $\mathrm{C}$ & $\mathrm{C} 2$ & ${ }^{*} \mathrm{~N}$ & $\mathrm{HN}$ & 1.0000 & 120.00 & 180.00 & 120.00 & 1.0000 \\
\hline IO & CT & $\mathrm{C}$ & $\mathrm{N}$ & $\mathrm{C} 2$ & 1.0000 & 120.00 & 180.00 & 120.00 & 1.000 \\
\hline 10 & $\mathrm{~N}$ & CT & $*^{*} \mathrm{C}$ & $\mathrm{O}$ & 1.0000 & 120.00 & 180.00 & 120.00 & 1.000 \\
\hline 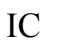 & $\mathrm{O}$ & $\mathrm{C}$ & $\mathrm{CT}$ & HT1 & 1.0000 & 120.00 & 180.00 & 120.00 & 1.0000 \\
\hline IC & $\mathrm{O}$ & $\mathrm{C}$ & 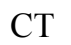 & HT2 & 1.0000 & 120.00 & 60.00 & 120.00 & 1.0000 \\
\hline IC & $\mathrm{O}$ & $\mathrm{C}$ & $\mathrm{CT}$ & HT3 & 1.0000 & 120.00 & -60.00 & 120.00 & 1.000 \\
\hline
\end{tabular}

PATCH FIRST NONE LAST NONE 


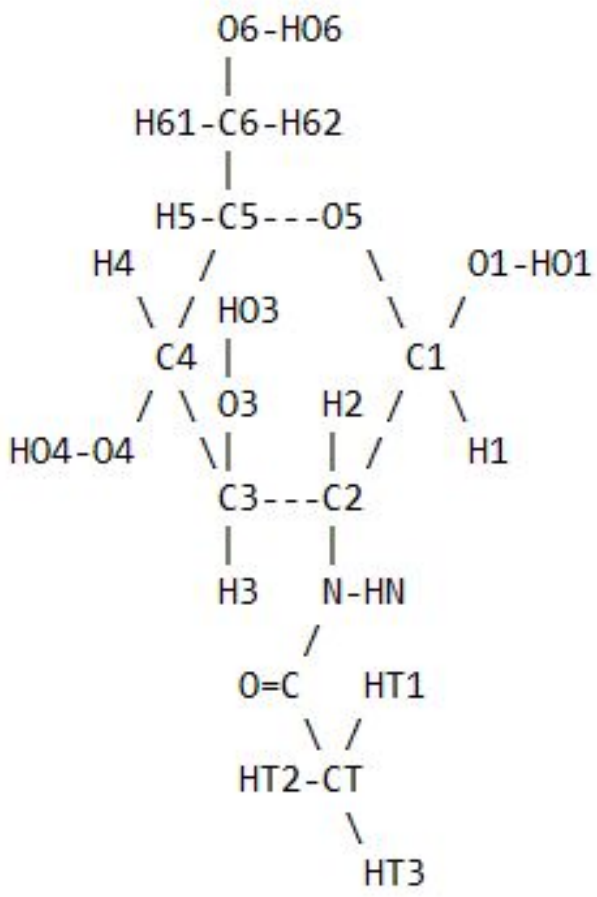

GROUP

ATOM C1

CD31HA

0.124 ALPHA -1.041 THOLE 2.120 !

ATOM H1

HDA1A

0.070

ATOM O1

OD31E

0.000 ALPHA -0.847 THOLE 0.571 !

ATOM HO1

HDP1A

0.333

ATOM C5

CD31HC

0.112 ALPHA -1.004 THOLE 0.723 !

ATOM H5

HDA2E

0.069

ATOM O5

OD306A

0.000 ALPHA -0.811 THOLE 0.407 !

ATOM LP1A

LPD

$-0.202$

ATOM LP1B

LPD

$-0.202$

$-0.152$

ATOM LP5B

LPD

LPD

ATOM LPX5

LPD

GROUP

ATOM C2

CD31HB

ATOM H2

HDA1A

$-0.152$

0.000 !dummy for anisotropic polarizability

ATOM N

ND2A2

0.082 ALPHA -1.090 THOLE 0.208

0.070

ATOM HN

GROUP

ATOM CT

HDP1A

-0.382 ALPHA -1.942 THOLE 0.250

0.230

ATOM HT1

CD33C

-0.102 ALPHA -1.894 THOLE 2.122

ATOM HT2

HDA3A

0.048

0.048

HDA3A

HDA3A

0.048

ATOM C

CD2O1A

0.447 ALPHA -0.675 THOLE 0.295

ATOM O OD2C1A

0.050 ALPHA -0.651 THOLE 0.310

ATOM LPY1

LPDO1

$-0.312$

ATOM LPY2

GROUP

ATOM C3

LPDO1

$-0.227$

ATOM H3

CD31HB

-0.006 ALPHA -1.007 THOLE 1.735

HDA1A

0.070

0.000 ALPHA -0.502 THOLE 0.929

ATOM HO3

D31E

ATOM LP3A

HDP1A

0.322

$-0.193$ 


$\begin{array}{llr}\text { ATOM LP3B } & \text { LPD } & -0.193 \\ \text { GROUP } & & -0.006 \text { ALPHA }-1.031 \text { THOLE } 0.552 \\ \text { ATOM C4 } & \text { CD31HB } & 0.070 \\ \text { ATOM H4 } & \text { HDA1A } & 0.000 \text { ALPHA -0.902 THOLE } 2.077 \\ \text { ATOM O4 } & \text { OD31E } & 0.322 \\ \text { ATOM HO4 } & \text { HDP1A } & -0.193 \\ \text { ATOM LP4A } & \text { LPD } & -0.193 \\ \text { ATOM LP4B } & \text { LPD } & \\ \text { GROUP } & & -0.086 \text { ALPHA -1.005 THOLE } 0.249 ! \\ \text { ATOM C6 } & \text { CD32A } & 0.080 \\ \text { ATOM H61 } & \text { HDA2A } & 0.080 \\ \text { ATOM H62 } & \text { HDA2A } & 0.000 \text { ALPHA -0.778 THOLE } 0.207 \text { ! } \\ \text { ATOM O6 } & \text { OD31A } & 0.328 \\ \text { ATOM HO6 } & \text { HDP1A } & -0.201 \\ \text { ATOM LP6A } & \text { LPD } & -0.201\end{array}$

LONEPAIR relative LP1A O1 C1 HO1 distance 0.35 angle 110.0 dihe 91.0 LONEPAIR relative LP1B O1 C1 HO1 distance 0.35 angle 110.0 dihe 269.0 ANISOTROPY O1 C1 LP1A LP1B A11 0.8108 A22 1.2162

LONEPAIR relative LP3A O3 C3 HO3 distance 0.35 angle 110.0 dihe 91.0 LONEPAIR relative LP3B O3 C3 HO3 distance 0.35 angle 110.0 dihe 269.0 ANISOTROPY O3 C3 LP3A LP3B A11 0.8108 A22 1.2162

LONEPAIR relative LP4A O4 C4 HO4 distance 0.35 angle 110.0 dihe 91.0 LONEPAIR relative LP4B O4 C4 HO4 distance 0.35 angle 110.0 dihe 269.0 ANISOTROPY O4 C4 LP4A LP4B A11 0.8108 A22 1.2162

LONEPAIR bisector LP5A O5 C1 C5 distance 0.35 angle 110.0 dihe 90.0 LONEPAIR bisector LP5B O5 C1 C5 distance 0.35 angle 110.0 dihe 270.0 LONEPAIR bisector LPX5 O5 C1 C5 distance 0.10 angle 0.0 dihe 0.0 ANISOTROPY O5 LPX5 LP5A LP5B A11 0.8889 A22 1.2222

LONEPAIR relative LP6A O6 C6 HO6 distance 0.35 angle 110.0 dihe 91.0 LONEPAIR relative LP6B O6 C6 HO6 distance 0.35 angle 110.0 dihe 269.0 ANISOTROPY O6 C6 LP6A LP6B A11 0.8108 A22 1.2162

LONEPAIR relative LPY1 O C CT distance 0.30 angle 91.0 dihe 0.0 LONEPAIR relative LPY2 O C CT distance 0.30 angle 91.0 dihe 180.0 ANISOTROPY O C LPY1 LPY2 A11 0.82322 A22 1.14332

$\begin{array}{llllllllll}\text { ! } & \mathrm{I} & \mathrm{J} & \mathrm{K} & \mathrm{L} & \mathrm{R}(\mathrm{IK}) & \mathrm{T}(\mathrm{IKJ}) & \mathrm{PHI} & \mathrm{T}(\mathrm{JKL}) & \mathrm{R}(\mathrm{KL}) \\ \mathrm{IC} & \mathrm{O} 1 & \mathrm{C} 2 & * \mathrm{C} 1 & \mathrm{H} 1 & 1.3949 & 109.50 & 118.29 & 110.11 & 1.1152 \\ \mathrm{IC} & \mathrm{O} 1 & \mathrm{O} 5 & * \mathrm{C} 1 & \mathrm{C} 2 & 1.3949 & 110.13 & 120.34 & 109.32 & 1.5156 \\ \mathrm{IC} & \mathrm{N} & \mathrm{C} 3 & * \mathrm{C} 2 & \mathrm{H} 2 & 1.4607 & 113.70 & -119.19 & 107.08 & 1.1227\end{array}$




\begin{tabular}{|c|c|c|c|c|c|c|c|c|c|}
\hline C & $\mathrm{N}$ & 1 & 22 & C3 & 1.4607 & 11262 & 2 & 109.20 & 1.5149 \\
\hline IC & $\mathrm{O} 3$ & C4 & ${ }^{*} \mathrm{C} 3$ & $\mathrm{H} 3$ & 1.4246 & 110.45 & 117.60 & 108.58 & 1.1171 \\
\hline$C$ & $\mathrm{O} 3$ & $\mathrm{C} 2$ & ${ }^{*} \mathrm{C} 3$ & $\mathrm{C} 4$ & 1.4246 & 111.13 & 123.24 & 110.86 & 1.5168 \\
\hline IC & $\mathrm{O} 4$ & C5 & ${ }^{*} \mathrm{C} 4$ & H4 & 1.4204 & 110.47 & -117.94 & 108.07 & 1.1172 \\
\hline W & $\mathrm{O} 4$ & C3 & ${ }^{*} \mathrm{C} 4$ & C5 & 1.4204 & & & 110.79 & \\
\hline IC & C6 & O5 & ${ }^{*} \mathrm{C} 5$ & H5 & 1.5134 & 108.06 & 117.57 & 109.86 & 1.1171 \\
\hline IC & C6 & $\mathrm{C} 4$ & ${ }^{*} \mathrm{C} 5$ & $\mathrm{O} 5$ & 1.5134 & 113.35 & 119.99 & 108.45 & 1.4386 \\
\hline IC & O6 1 & H62 & ${ }^{*} \mathrm{C} 6$ & H61 & 1.4280 & 109.26 & -117.58 & 107.87 & 1.1141 \\
\hline IC & O6 & C5 & *C6 I & H62 & 1.4280 & 111.18 & -121.26 & 110.14 & 1.1132 \\
\hline IC & O5 & $\mathrm{C} 1$ & $\mathrm{C} 2$ & $\mathrm{C} 3$ & 1.4220 & 109.32 & 58.90 & 109.20 & 1.5149 \\
\hline IC & $\mathrm{C} 1$ & $\mathrm{C} 2$ & C3 & $\mathrm{C} 4$ & 1.5156 & 109.20 & -52.68 & 110.86 & 1.5168 \\
\hline IC & $\mathrm{C} 2$ & C3 & $\mathrm{C} 4$ & C5 & 1.5149 & 110.86 & 52.13 & 110.79 & 1.5206 \\
\hline IC & $\mathrm{C} 3$ & $\mathrm{C} 4$ & C5 & $\mathrm{O} 5$ & 1.5168 & 110.79 & -56.10 & 108.45 & 1.4386 \\
\hline IC & $\mathrm{C} 4$ & C5 & O5 & $\mathrm{C} 1$ & 1.5206 & 108.45 & 64.09 & 111.47 & 1.4220 \\
\hline IC & C5 & O5 & $\mathrm{C} 1$ & $\mathrm{C} 2$ & 1.4386 & 111.47 & -66.18 & 109.32 & 1.5156 \\
\hline IC & $\mathrm{C} 4$ & C5 & C6 & O6 & 1.5206 & 113.35 & -179.21 & 111.18 & 1.4280 \\
\hline IC & O5 & $\mathrm{C} 1$ & O1 & HO1 & 1.4220 & 110.13 & 53.79 & 107.03 & 0.9601 \\
\hline IC & $\mathrm{C} 1$ & $\mathrm{C} 2$ & $\mathrm{~N}$ & $\mathrm{HN}$ & 1.5156 & 112.62 & -21.73 & 117.18 & 0.9940 \\
\hline IC & $\mathrm{C} 2$ & $\mathrm{C} 3$ & O3 I & $\mathrm{HO} 3$ & 1.5149 & 111.13 & 0.20 & 109.43 & 0.9762 \\
\hline IC & C3 & $\mathrm{C} 4$ & O4 I & $\mathrm{HO} 4$ & 1.5168 & 110.88 & 45.93 & 106.90 & 0.9672 \\
\hline I & C5 & C6 & O6 I & HO6 & 1.5134 & 111.18 & -58.35 & 108.74 & 0.9641 \\
\hline IC & $\mathrm{C}$ & $\mathrm{N}$ & $\mathrm{C} 2$ & C3 & 1.3365 & 123.04 & -84.98 & 113.70 & 1.5149 \\
\hline IC & $\mathrm{C}$ & $\mathrm{C} 2$ & $* \mathrm{~N}$ & $\mathrm{HN}$ & 1.3365 & 123.04 & -171.8 & 117.18 & 0.9940 \\
\hline IC & CT & $\mathrm{C}$ & $\mathrm{N}$ & $\mathrm{C} 2$ & 1.4798 & 117.02 & -173 & 123.04 & 1.4607 \\
\hline IC & $\mathrm{N}$ & CT & ${ }^{*} \mathrm{C}$ & $\mathrm{O}$ & 1.3365 & 117.02 & 178.97 & 121.63 & 1.2235 \\
\hline IC & $\mathrm{O}$ & $\mathrm{C}$ & CT & HT1 & 1.2235 & 121.63 & 116.39 & 110.28 & 1.1105 \\
\hline IC & $\mathrm{O}$ & $\mathrm{C}$ & CT & HT2 & 1.2235 & 121.63 & -3.06 & 109.29 & 1.1121 \\
\hline IC & $\mathrm{O}$ & $\mathrm{C}$ & $\mathrm{C}^{\prime}$ & HT3 & 1.2235 & 121.63 & -122.59 & 110.33 & 1.1105 \\
\hline
\end{tabular}

PATCH FIRST NONE LAST NONE

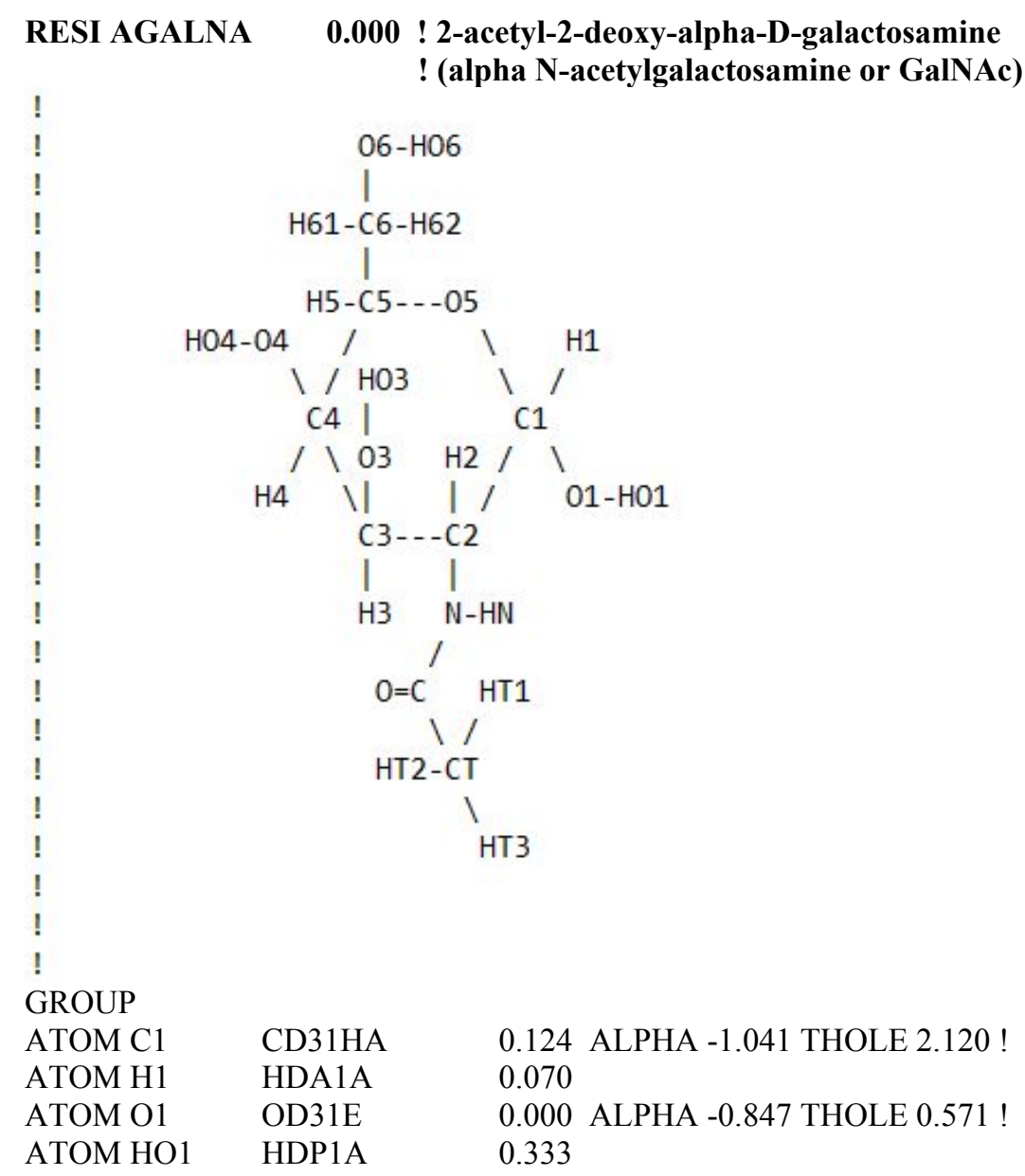




\begin{tabular}{|c|c|c|c|c|}
\hline ATOM C5 & $\mathrm{CD} 31 \mathrm{HC}$ & 0.112 & ALPHA -1.004 THOLE $0.723 !$ & \\
\hline ATOM H5 & HDA2E & 0.069 & & \\
\hline ATOM O5 & OD306A & 0.000 & ALPHA -0.811 THOLE 0.407 ! & \\
\hline ATOM LP1A & LPD & -0.202 & & \\
\hline ATOM LP1B & LPD & -0.202 & & \\
\hline ATOM LP5A & LPD & -0.152 & & \\
\hline ATOM LP5B & LPD & -0.152 & & \\
\hline ATOM LPX5 & LPD & 0.000 & !dummy for anisotropic polarizability & \\
\hline \multicolumn{5}{|c|}{ 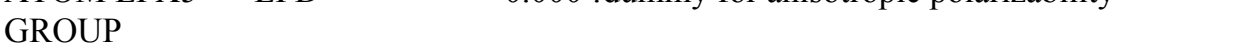 } \\
\hline ATOM C2 & CD31HB & 0.082 & ALPHA - 1.090 THOLE 0.208 & \\
\hline ATOM H2 & HDA1A & 0.070 & & \\
\hline ATOM N & ND2A2 & -0.382 & ALPHA -1.942 THOLE 0.250 & \\
\hline ATOM HN & HDP1A & 0.230 & & \\
\hline \multicolumn{5}{|l|}{ GROUP } \\
\hline ATOM CT & CD33C & -0.102 & ALPHA -1.894 THOLE 2.122 & \\
\hline ATOM HT1 & HDA3A & 0.048 & & \\
\hline ATOM HT2 & HDA3A & 0.048 & & \\
\hline ATOM HT3 & HDA3A & 0.048 & & \\
\hline ATOM C & $\mathrm{CD} 2 \mathrm{O} 1 \mathrm{~A}$ & 0.447 & ALPHA -0.675 THOLE 0.295 & \\
\hline ATOM O & $\mathrm{OD} 2 \mathrm{C} 1 \mathrm{~A}$ & 0.050 & ALPHA -0.651 THOLE 0.310 & \\
\hline ATOM LPY1 & LPDO1 & -0.312 & & \\
\hline ATOM LPY2 & LPDO1 & -0.227 & & \\
\hline \multicolumn{5}{|l|}{ GROUP } \\
\hline ATOM C3 & CD31HB & -0.006 & ALPHA - 1.007 THOLE 1.735 & \\
\hline АTOM H3 & HDA1A & 0.070 & & \\
\hline ATOM O3 & OD31E & 0.000 & ALPHA -0.502 THOLE 0.929 & \\
\hline ATOM HO3 & HDP1A & 0.322 & & \\
\hline ATOM LP3A & LPD & -0.193 & & \\
\hline ATOM LP3B & LPD & -0.193 & & \\
\hline \multicolumn{5}{|l|}{ GROUP } \\
\hline ATOM C4 & CD31HB & -0.006 & ALPHA -1.031 THOLE 0.552 & \\
\hline ATOM H4 & HDA1A & 0.070 & & \\
\hline ATOM O4 & OD31E & 0.000 & ALPHA -0.902 THOLE 2.077 & \\
\hline ATOM HO4 & HDP1A & 0.322 & & \\
\hline ATOM LP4A & LPD & -0.193 & & \\
\hline ATOM LP4B & LPD & -0.193 & & \\
\hline \multicolumn{5}{|l|}{ GROUP } \\
\hline ATOM C6 & CD32A & -0.086 & ALPHA -1.005 THOLE 0.249 ! & \\
\hline ATOM H61 & $\mathrm{HDA} 2 \mathrm{~A}$ & 0.080 & & \\
\hline ATOM H62 & HDA2A & 0.080 & & \\
\hline ATOM O6 & OD31A & 0.000 & ALPHA -0.778 THOLE 0.207 ! & \\
\hline ATOM HO6 & HDP1A & 0.328 & & \\
\hline ATOM LP6A & LPD & -0.201 & & \\
\hline \multicolumn{5}{|l|}{ ! } \\
\hline BOND C1 O1 & & $\mathrm{C} 1 \mathrm{H} 1$ & O1 HO1 & $\mathrm{C} 1 \mathrm{C} 2$ \\
\hline BOND C2 H2 & & $\mathrm{C} 2 \mathrm{~N}$ & $\mathrm{~N} \quad \mathrm{HN}$ & $\mathrm{C} 3 \mathrm{H} 3$ \\
\hline BOND C3 O3 & & $\mathrm{O} 3 \mathrm{HO} 3$ & $\mathrm{C} 4 \mathrm{H} 4$ & $\mathrm{C} 4 \mathrm{O} 4$ \\
\hline BOND O4 HO4 & & $\mathrm{C} 4 \mathrm{C} 5$ & $\mathrm{C} 5 \mathrm{H} 5$ & C6 H61 \\
\hline BOND C6 H62 & & C6 O6 & O6 HO6 & $\mathrm{N} \quad \mathrm{C}$ \\
\hline BOND C O & & $\mathrm{C} \mathrm{CT}$ & CT HT1 & $\mathrm{CT} \mathrm{HT} 3$ \\
\hline BOND O1 LP1A & & O1 LP1B & & \\
\hline BOND O3 LP3A & & O3 LP3B & & \\
\hline BOND O4 LP4A & & O4 LP4B & & \\
\hline BOND O5 LP5A & & O5 LP5B & O5 LPX5 & \\
\hline BOND O6 LP6A & & O6 LP6B & & \\
\hline BOND O LPY1 & & O LPY2 & & \\
\hline IMPR C CT N O & & & & \\
\hline IMPR N C C2 H & & & & \\
\hline LONEPAIR relat & & $\mathrm{C} 1 \mathrm{HO}$ & 0 & \\
\hline
\end{tabular}


LONEPAIR relative LP1B O1 C1 HO1 distance 0.35 angle 110.0 dihe 269.0 ANISOTROPY O1 C1 LP1A LP1B A11 0.8108 A22 1.2162

LONEPAIR relative LP3A O3 C3 HO3 distance 0.35 angle 110.0 dihe 91.0 LONEPAIR relative LP3B O3 C3 HO3 distance 0.35 angle 110.0 dihe 269.0 ANISOTROPY O3 C3 LP3A LP3B A11 0.8108 A22 1.2162

LONEPAIR relative LP4A O4 C4 HO4 distance 0.35 angle 110.0 dihe 91.0 LONEPAIR relative LP4B O4 C4 HO4 distance 0.35 angle 110.0 dihe 269.0 ANISOTROPY O4 C4 LP4A LP4B A11 0.8108 A22 1.2162

LONEPAIR bisector LP5A O5 C1 C5 distance 0.35 angle 110.0 dihe 90.0 LONEPAIR bisector LP5B O5 C1 C5 distance 0.35 angle 110.0 dihe 270.0 LONEPAIR bisector LPX5 O5 C1 C5 distance 0.10 angle 0.0 dihe 0.0 ANISOTROPY O5 LPX5 LP5A LP5B A11 0.8889 A22 1.2222

LONEPAIR relative LP6A O6 C6 HO6 distance 0.35 angle 110.0 dihe 91.0 LONEPAIR relative LP6B O6 C6 HO6 distance 0.35 angle 110.0 dihe 269.0 ANISOTROPY O6 C6 LP6A LP6B A11 0.8108 A22 1.2162

LONEPAIR relative LPY1 O C CT distance 0.30 angle 91.0 dihe 0.0 LONEPAIR relative LPY2 O C CT distance 0.30 angle 91.0 dihe 180.0 ANISOTROPY O C LPY1 LPY2 A11 0.82322 A22 1.14332

\begin{tabular}{|c|c|c|c|c|c|c|c|c|c|}
\hline ! & I & $\mathrm{J}$ & $\mathrm{K}$ & $\mathrm{L}$ & $\mathrm{R}(\mathrm{IK})$ & $\mathrm{T}(\mathrm{IKJ})$ & PHI & $\mathrm{T}(\mathrm{JKL})$ & $\mathrm{R}(\mathrm{KL})$ \\
\hline IC & $\mathrm{O} 1$ & $\mathrm{C} 2$ & ${ }^{*} \mathrm{C} 1$ & H1 & 1.4006 & 109.41 & -118.16 & 109.00 & 1.1146 \\
\hline IC & $\mathrm{O} 1$ & $\mathrm{O5}$ & $* \mathrm{C} 1$ & $\mathrm{C} 2$ & 1.4006 & 112.45 & -122.23 & 110.03 & 1.5235 \\
\hline $\mathrm{IC}$ & $\mathrm{N}$ & $\mathrm{C} 3$ & $* \mathrm{C} 2$ & $\mathrm{H} 2$ & 1.4542 & 11 & 5.73 & 107.43 & 1.1188 \\
\hline IC & $\mathrm{N}$ & $\mathrm{C} 1$ & $* \mathrm{C} 2$ & $\mathrm{C} 3$ & 1.4542 & $115.05-$ & -126.93 & 109.87 & 1.5127 \\
\hline IC & $\mathrm{O} 3$ & $\mathrm{C} 4$ & ${ }^{*} \mathrm{C} 3$ & H3 & 1.4221 & 111.39 & 117.56 & 108.59 & 1.1168 \\
\hline IC & $\mathrm{O} 3$ & $\mathrm{C} 2$ & ${ }^{*} \mathrm{C} 3$ & $\mathrm{C} 4$ & 1.4221 & 110.78 & 124.12 & 110.75 & 1.5146 \\
\hline $\mathrm{IC}$ & $\mathrm{O} 4$ & C5 & ${ }^{*} \mathrm{C} 4$ & $\mathrm{H} 4$ & 1.4265 & 110.79 & 117.97 & 108.77 & 1.1167 \\
\hline IC & $\mathrm{O} 4$ & C3 & ${ }^{*} \mathrm{C} 4$ & $\mathrm{C} 5$ & 1.4265 & 111.93 & 123.14 & 109.33 & 1.5203 \\
\hline IC & C6 & O5 & $* \mathrm{C} 5$ & H5 & 1.5146 & 108.49 & 117.15 & 0.05 & 1.1177 \\
\hline IC & C6 & $\mathrm{C} 4$ & ${ }^{*} \mathrm{C} 5$ & O5 & 1.5146 & 113.21 & 121.21 & 109.51 & 1.4431 \\
\hline $\mathrm{IC}$ & O6 & H62 & *C6 & H61 & 1.4272 & 109.21 & -117.35 & 107.74 & 1.1138 \\
\hline IC & O6 & C5 & *C6 & H62 & 1.4272 & 111.21 & -121.61 & 110.72 & 1.1131 \\
\hline IC & $\mathrm{O} 5$ & $\mathrm{Cl}$ & $\mathrm{C} 2$ & $\mathrm{C} 3$ & 1.4328 & 110.03 & 55.96 & 109.87 & 1.5127 \\
\hline IC & $\mathrm{C} 1$ & $\mathrm{C} 2$ & $\mathrm{C} 3$ & $\mathrm{C} 4$ & 1.5 & 109.87 & -54.38 & .75 & 1.5146 \\
\hline IC & $\mathrm{C} 2$ & C3 & $\mathrm{C} 4$ & $\mathrm{C} 5$ & 1.5127 & 110.75 & 55.35 & 109.33 & 1.5203 \\
\hline $\mathrm{IC}$ & $\mathrm{C} 3$ & $\mathrm{C} 4$ & $\mathrm{C} 5$ & O5 & 1.5146 & 109.33 & -57.71 & 109.51 & 1.4431 \\
\hline IC & $\mathrm{C} 4$ & $\mathrm{C} 5$ & O5 & $\mathrm{C} 1$ & 1.5203 & 109.51 & 61.91 & 112.69 & 1.4328 \\
\hline IC & $\mathrm{C} 5$ & O5 & $\mathrm{C} 1$ & $\mathrm{C} 2$ & 1.4431 & 112.69 & -60.89 & 110.03 & 1.5235 \\
\hline IC & $\mathrm{C} 4$ & $\mathrm{C} 5$ & C6 & O6 & 1.5203 & 113.21 & -172.44 & 111.21 & 1.4272 \\
\hline $\mathrm{IC}$ & O5 & $\mathrm{C} 1$ & $\mathrm{O} 1$ & $\mathrm{HO} 1$ & 1.4328 & 112.45 & 72.07 & 107.32 & 0.9601 \\
\hline IC & $\mathrm{C} 1$ & $\mathrm{C} 2$ & $\mathrm{~N}$ & $\mathrm{HN}$ & 1.5235 & 115.05 & -118.24 & 115.62 & 0.9937 \\
\hline IC & $\mathrm{C} 2$ & C3 & $\mathrm{O} 3$ & $\mathrm{HO} 3$ & 1.5127 & 110.78 & 86.08 & 107.29 & 0.9662 \\
\hline IC & $\mathrm{C} 3$ & $\mathrm{C} 4$ & $\mathrm{O} 4$ & $\mathrm{HO}$ & 1.5146 & 111.93 & 81.54 & 108.79 & 0.9647 \\
\hline $\mathrm{IC}$ & $\mathrm{C} 5$ & C6 & O6 & HO6 & 1.5146 & 111.21 & -63.87 & 109.07 & 0.9637 \\
\hline IC & $\mathrm{C}$ & $\mathrm{N}$ & $\mathrm{C} 2$ & $\mathrm{C} 3$ & 1.3354 & 123.56 & -169.15 & 111.59 & 1.5127 \\
\hline IC & $\mathrm{C}$ & $\mathrm{C} 2$ & $* \mathrm{~N}$ & $\mathrm{HN}$ & 1.3354 & 123.56 & 176.96 & 115.62 & 0.9937 \\
\hline IC & $\mathrm{CT}$ & $\mathrm{C}$ & $\mathrm{N}$ & $\mathrm{C} 2$ & 1.4804 & 116.54 & 179.29 & 123.56 & 1.4542 \\
\hline IC & $\mathrm{N}$ & $\mathrm{CT}$ & $* \mathrm{C}$ & $\mathrm{O}$ & 1.3354 & 116.54 & 179.78 & 121.19 & 1.2233 \\
\hline $\mathrm{IC}$ & $\mathrm{O}$ & $\mathrm{C}$ & $\mathrm{CT}$ & HT1 & 1.2233 & 121.19 & 119.84 & 110.19 & 1.1104 \\
\hline IC & $\mathrm{O}$ & $\mathrm{C}$ & $\mathrm{CT}$ & HT2 & 1.2233 & 121.19 & 0.28 & 109.29 & 1.1117 \\
\hline IC & $\mathrm{O}$ & $\mathrm{C}$ & $\mathrm{CT}$ & HT3 & 1.2233 & 121.19 & -119.29 & 110.17 & 1.1104 \\
\hline
\end{tabular}
PATCH FIRST NONE LAST NONE

RESI BGALNA $\quad 0.000 ! 2$-acetyl-2-deoxy-beta-D-galactosamine ! (beta N-acetylgalactosamine or GalNAc) 


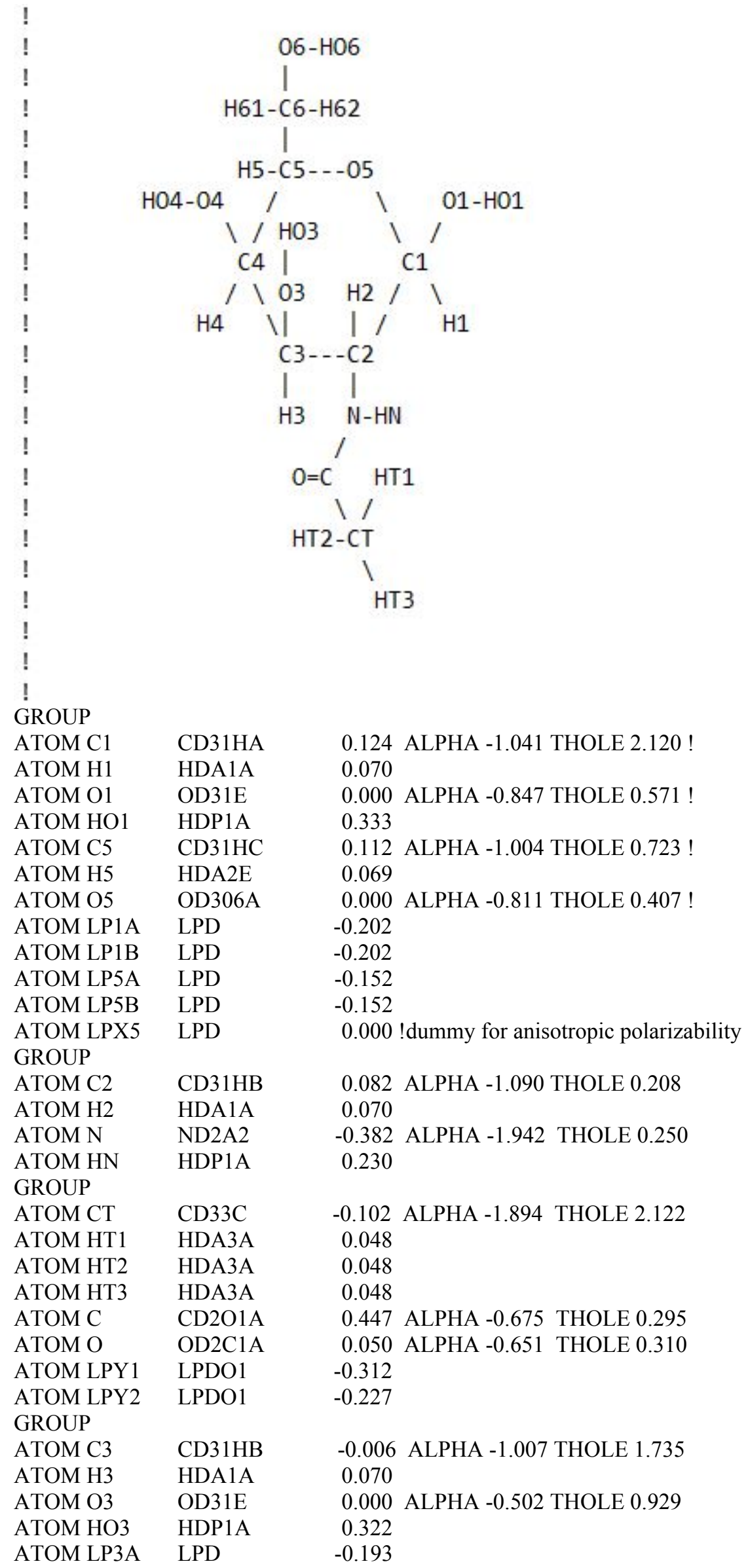




$\begin{array}{llr}\text { ATOM LP3B } & \text { LPD } & -0.193 \\ \text { GROUP } & & -0.006 \text { ALPHA }-1.031 \text { THOLE } 0.552 \\ \text { ATOM C4 } & \text { CD31HB } & 0.070 \\ \text { ATOM H4 } & \text { HDA1A } & 0.000 \text { ALPHA -0.902 THOLE } 2.077 \\ \text { ATOM O4 } & \text { OD31E } & 0.322 \\ \text { ATOM HO4 } & \text { HDP1A } & -0.193 \\ \text { ATOM LP4A } & \text { LPD } & -0.193 \\ \text { ATOM LP4B } & \text { LPD } & \\ \text { GROUP } & & -0.086 \text { ALPHA -1.005 THOLE } 0.249 ! \\ \text { ATOM C6 } & \text { CD32A } & 0.080 \\ \text { ATOM H61 } & \text { HDA2A } & 0.080 \\ \text { ATOM H62 } & \text { HDA2A } & 0.000 \text { ALPHA -0.778 THOLE } 0.207 \text { ! } \\ \text { ATOM O6 } & \text { OD31A } & 0.328 \\ \text { ATOM HO6 } & \text { HDP1A } & -0.201 \\ \text { ATOM LP6A } & \text { LPD } & -0.201\end{array}$

LONEPAIR relative LP3A O3 C3 HO3 distance 0.35 angle 110.0 dihe 91.0 LONEPAIR relative LP3B O3 C3 HO3 distance 0.35 angle 110.0 dihe 269.0 ANISOTROPY O3 C3 LP3A LP3B A11 0.8108 A22 1.2162

LONEPAIR relative LP4A O4 C4 HO4 distance 0.35 angle 110.0 dihe 91.0 LONEPAIR relative LP4B O4 C4 HO4 distance 0.35 angle 110.0 dihe 269.0 ANISOTROPY O4 C4 LP4A LP4B A11 0.8108 A22 1.2162

LONEPAIR bisector LP5A O5 C1 C5 distance 0.35 angle 110.0 dihe 90.0 LONEPAIR bisector LP5B O5 C1 C5 distance 0.35 angle 110.0 dihe 270.0 LONEPAIR bisector LPX5 O5 C1 C5 distance 0.10 angle 0.0 dihe 0.0 ANISOTROPY O5 LPX5 LP5A LP5B A11 0.8889 A22 1.2222

LONEPAIR relative LP6A O6 C6 HO6 distance 0.35 angle 110.0 dihe 91.0 LONEPAIR relative LP6B O6 C6 HO6 distance 0.35 angle 110.0 dihe 269.0 ANISOTROPY O6 C6 LP6A LP6B A11 0.8108 A22 1.2162

LONEPAIR relative LPY1 O C CT distance 0.30 angle 91.0 dihe 0.0 LONEPAIR relative LPY2 O C CT distance 0.30 angle 91.0 dihe 180.0 ANISOTROPY O C LPY1 LPY2 A11 0.82322 A22 1.14332

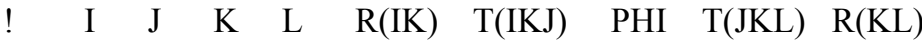

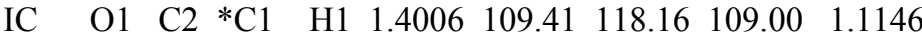
$\begin{array}{llllllllll}\mathrm{IC} & \mathrm{O} 1 & \mathrm{O} 5 & * \mathrm{C} 1 & \mathrm{C} 2 & 1.4006 & 112.45 & 122.23 & 110.03 & 1.5235\end{array}$
$\begin{array}{llllllllll}\mathrm{IC} & \mathrm{N} & \mathrm{C} 3 & * \mathrm{C} 2 & \mathrm{H} 2 & 1.4542 & 111.59 & -115.73 & 107.43 & 1.1188\end{array}$ 


\begin{tabular}{|c|c|c|c|c|c|c|c|c|c|}
\hline IC & $\mathrm{N}$ & $\mathrm{C} 1$ & $* \mathrm{C} 2$ & $\mathrm{C} 3$ & 42 & 115.05 & 6.93 & 37 & 51 \\
\hline IC & $\mathrm{O} 3$ & $\mathrm{C} 4$ & $* \mathrm{C} 3$ & H3 & 1.4221 & 111.39 & 117.56 & 108.59 & 1.1168 \\
\hline $\mathrm{IC}$ & $\mathrm{O} 3$ & $\mathrm{C} 2$ & $* \mathrm{C} 3$ & $\mathrm{C} 4$ & 1.4221 & 110.78 & 124.12 & 110.75 & 1.5146 \\
\hline IC & O4 & $\mathrm{C} 5$ & $* \mathrm{C} 4$ & H4 & 1.4265 & 110.79 & 117.97 & 108.77 & 167 \\
\hline IC & $\mathrm{O} 4$ & $\mathrm{C} 3$ & $* \mathrm{C} 4$ & $\mathrm{C} 5$ & 1.4265 & 111.93 & 123.14 & 109.33 & 1.5203 \\
\hline IC & C6 & O5 & $* \mathrm{C} 5$ & H5 & 1.5146 & 108.49 & 117.15 & 110.05 & 1.1177 \\
\hline IC & C6 & $\mathrm{C} 4$ & *C5 & O5 & 1.5146 & 113.21 & 121.21 & 109.51 & 1.4431 \\
\hline $\mathrm{IC}$ & O6 & H62 & *C6 & H61 & 1.4272 & 10 & -117.35 & 107.74 & 1.1138 \\
\hline $\mathrm{IC}$ & O6 & $\mathrm{C} 5$ & $* \mathrm{C} 6$ & H62 & 1.4272 & 11 & -121.61 & 110.72 & 1.1131 \\
\hline IC & O5 & $\mathrm{C} 1$ & $\mathrm{C} 2$ & $\mathrm{C} 3$ & 1.4328 & 03 & 55.96 & 10 & \\
\hline $\mathrm{IC}$ & $\mathrm{C} 1$ & $\mathrm{C} 2$ & $\mathrm{C} 3$ & $\mathrm{C} 4$ & 1.5235 & 109.87 & -54.38 & 110.75 & 1.5146 \\
\hline $\mathrm{IC}$ & $\mathrm{C} 2$ & C3 & $\mathrm{C} 4$ & $\mathrm{C} 5$ & .5127 & 11 & 55.35 & 10 & 1.5203 \\
\hline $\mathrm{IC}$ & C3 & $\mathrm{C} 4$ & C5 & O5 & 1.5146 & 33 & -57.71 & 10 & 431 \\
\hline IC & $\mathrm{C} 4$ & C5 & $\mathrm{O} 5$ & $\mathrm{C} 1$ & 1.5203 & 109.51 & 61.91 & 112.69 & 1.4328 \\
\hline $\mathrm{IC}$ & $\mathrm{C} 5$ & O5 & $\mathrm{C} 1$ & $\mathrm{C} 2$ & 1.4431 & 112.69 & -60.89 & 03 & 1.5235 \\
\hline IC & $\mathrm{C} 4$ & C5 & C6 & O6 & 1.5203 & 113.21 & -172.44 & 111.21 & 1.4272 \\
\hline IC & O5 & C1 & $\mathrm{O}$ & $\mathrm{HO} 1$ & 1.4328 & .45 & 125.25 & 107.32 & 0.9601 \\
\hline IC & $\mathrm{C} 1$ & $\mathrm{C} 2$ & $\mathrm{~N}$ & $\mathrm{HN}$ & 1.5235 & 115.05 & -118.24 & 115.62 & 0.9937 \\
\hline IC & $\mathrm{C} 2$ & C3 & $\mathrm{O} 3$ & HO3 & 1.5127 & 110.78 & 86.08 & 107.29 & 0.9662 \\
\hline IC & C3 & C4 & $\mathrm{O} 4$ & $\mathrm{HO} 4$ & 1.5146 & 111.93 & 81.54 & 108.79 & 0.9647 \\
\hline $\mathrm{IC}$ & $\mathrm{C} 5$ & C6 & O6 & HO6 & 1.5146 & 111.21 & -63.87 & 109.07 & 0.9637 \\
\hline IC & $\mathrm{C}$ & $\mathrm{N}$ & $\mathrm{C} 2$ & $\mathrm{C} 3$ & 1.3354 & 123.56 & -169.15 & 111.59 & 1.5127 \\
\hline $\mathrm{IC}$ & $\mathrm{C}$ & $\mathrm{C} 2$ & $* \mathrm{~N}$ & $\mathrm{HN}$ & 1.3354 & 123.56 & 176.96 & 115.62 & 0.9937 \\
\hline IC & CT & $\mathrm{C}$ & $\mathrm{N}$ & $\mathrm{C} 2$ & 1.4804 & 116.54 & 179.29 & 123.56 & 1.4542 \\
\hline IC & $\mathrm{N}$ & CT & $* \mathrm{C}$ & $\mathrm{O}$ & 1.3354 & 116.54 & 179.78 & 121.19 & 1.2233 \\
\hline IC & $\mathrm{O}$ & $\mathrm{C}$ & CT & HT1 & 1.2233 & 121.19 & 9119.84 & 110.19 & 1.1104 \\
\hline IC & $\mathrm{O}$ & $\mathrm{C}$ & $\mathrm{CT}$ & HT2 & 1.2233 & 121.19 & 0.28 & 109.29 & 1.1117 \\
\hline IC & $\mathrm{O}$ & $\mathrm{C}$ & CT & HT3 & 1.223 & 121.19 & $9-119.2$ & 110.17 & 1.1104 \\
\hline
\end{tabular}
PATCH FIRST NONE LAST NONE end

read para card append

* Drude polarizable FF parameters

*

BONDS

$\begin{array}{llll}\mathrm{CD} 31 \mathrm{HC} & \mathrm{CD} 2 \mathrm{O} 2 \mathrm{~A} & 225.00 & 1.538 \\ \mathrm{CD} 31 \mathrm{HB} & \mathrm{ND} 2 \mathrm{~A} 2 & 307.90 & 1.445\end{array}$

ANGLES

HDA2E

OD306A

CD31HB

CD31HC

CD31HA

HDA1A

ND2A2

CD31HB

CD31HB

CD31HC
CD31HC
CD31HC
CD2O2A
CD31HB
CD31HB
CD31HB
ND2A2
ND2A2

CD2O2A
CD2O2A
CD2O2A
OD2C2A
ND2A2
ND2A2
CD31HB
HDP1A
CD2O1A


OD306A
OD306A
OD306A
OD306A
CD2O2A
CD31HC
CD31HC
CD31HC
CD31HC

34.50

$110.10 \quad 22.532 .179$

$45.00 \quad 103.00$

$\begin{array}{lll}53.35 & 114.00 & 8.002 .561\end{array}$

DIHEDRALS

CD2O2A

CD31HC

40.00

109.30

$71.70 \quad 118.00$

$34.80 \quad 107.70$

$71.70 \quad 118.00$

$72.20 \quad 123.64$

$40.90 \quad 116.10$

$\begin{array}{lll}\text { CD2O2A } & \text { CD31HC } & \text { OD306A } \\ \text { CD2O2A } & \text { CD31HC } & \text { OD306A } \\ \text { CD2O2A } & \text { CD31HC } & \text { OD306A } \\ \text { HDA2E } & \text { CD31HC } & \text { CD2O2A } \\ \text { OD2C2A } & \text { CD2O2A } & \text { CD31HC } \\ \text { OD2C2A } & \text { CD2O2A } & \text { CD31HC } \\ \text { OD2C2A } & \text { CD2O2A } & \text { CD31HC } \\ \text { OD2C2A } & \text { CD2O2A } & \text { CD31HC }\end{array}$

CD31HA
CD31HA
CD31HA
CD31HA
OD2C2A
OD306A
OD306A
OD306A
OD306A

$\begin{array}{lll}0.1626 & 1 & 180.00\end{array}$ $\begin{array}{lll}0.8994 & 2 & 0.00\end{array}$ $\begin{array}{lll}1.5986 & 3 & 180.00\end{array}$ $\begin{array}{lll}0.4011 & 6 & 0.00\end{array}$ $\begin{array}{lll}0.0500 & 6 & 180.00\end{array}$ $\begin{array}{llll}0.0204 & 1 & 180.00\end{array}$ $\begin{array}{lll}0.7985 & 2 & 180.00\end{array}$ $\begin{array}{lll}0.7131 & 3 & 0.00\end{array}$ $\begin{array}{lll}0.1258 & 6 & 0.00\end{array}$ 


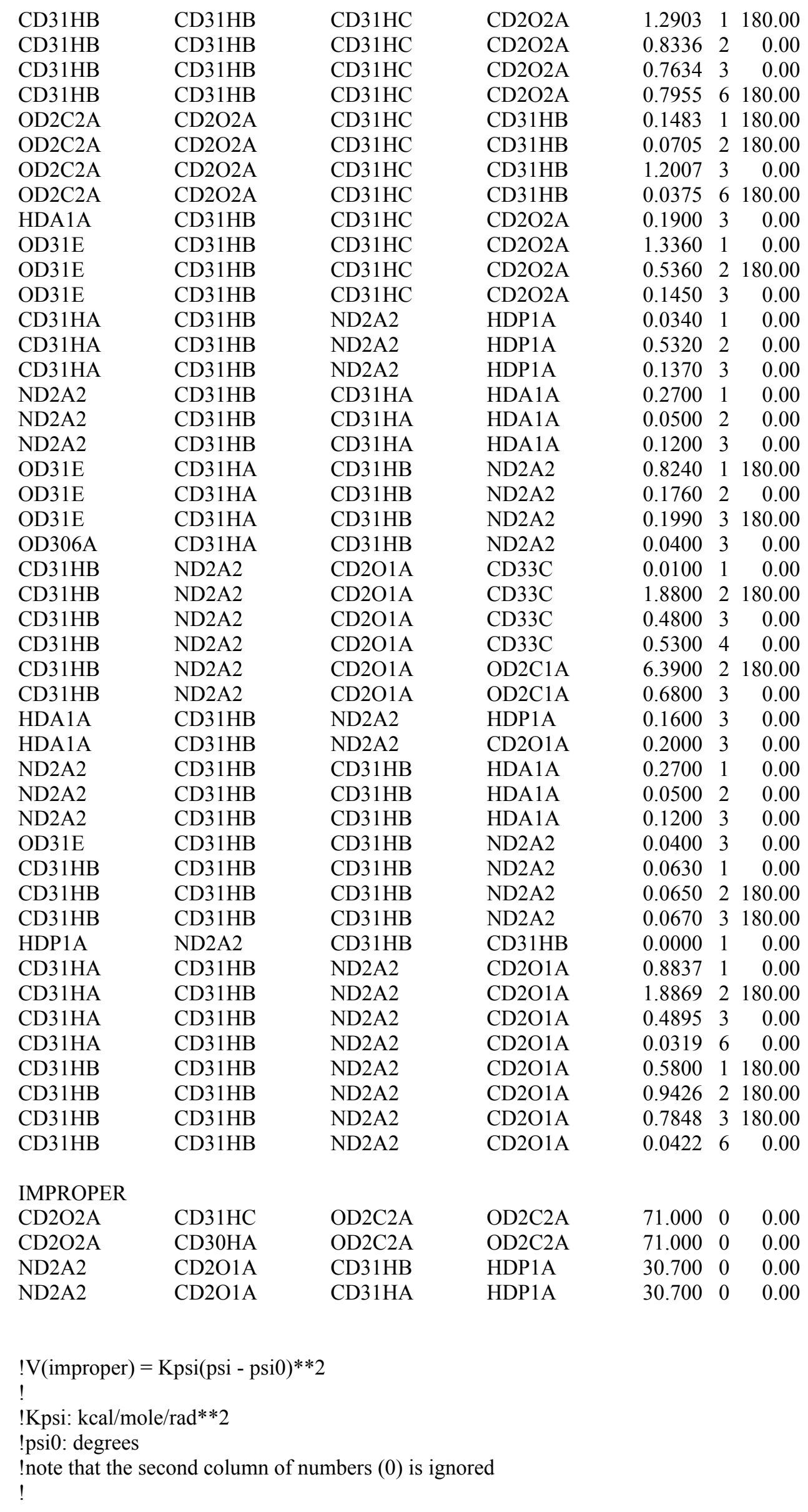


!dummy parameter for format

!CD2O1D CD32F CD31G OD2C1D $00.000 \quad 0 \quad 0.00$ !

NONBONDED nbxmod 5 atom vatom cdiel vdistance switch vswitch cutnb 16.0 ctofnb 12.0 ctonnb 10.0 eps 1.0 e14fac 1.0 wmin 1.5

!NBFIX

END 

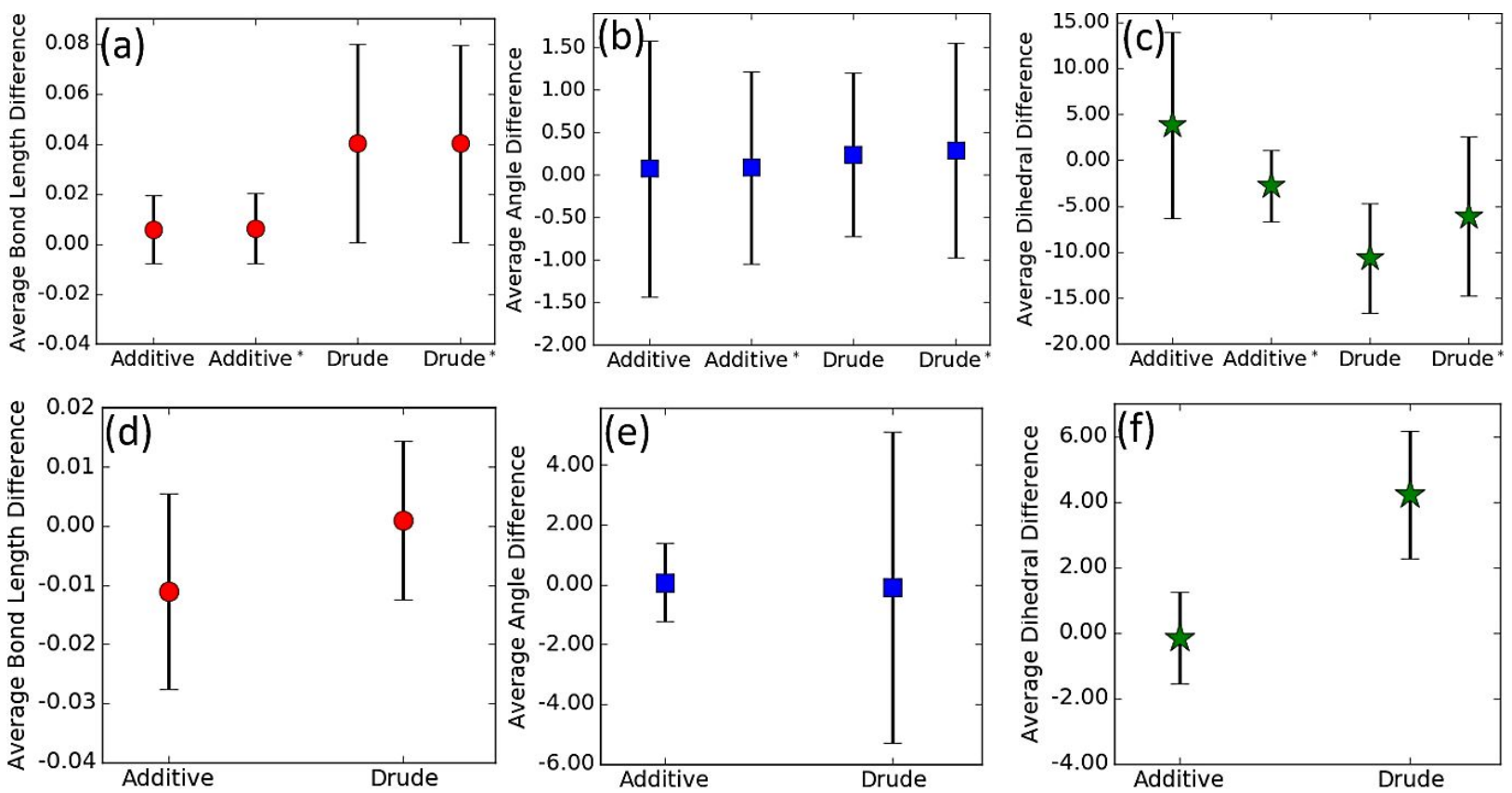

( ${ }^{*}$ restraining potential applied to the dihedral C1-O5-C5-C6 to maintain QM geometry.)

Figure S1. Figure showing average and standard deviation (represented as error bar) for MM - QM values of Bond Lengths (for model compound (a) M1, (d) M2); Angles (for model compound (b) M1, (e) M2); and Dihedrals (for model compound (c) M1, (f) M2).
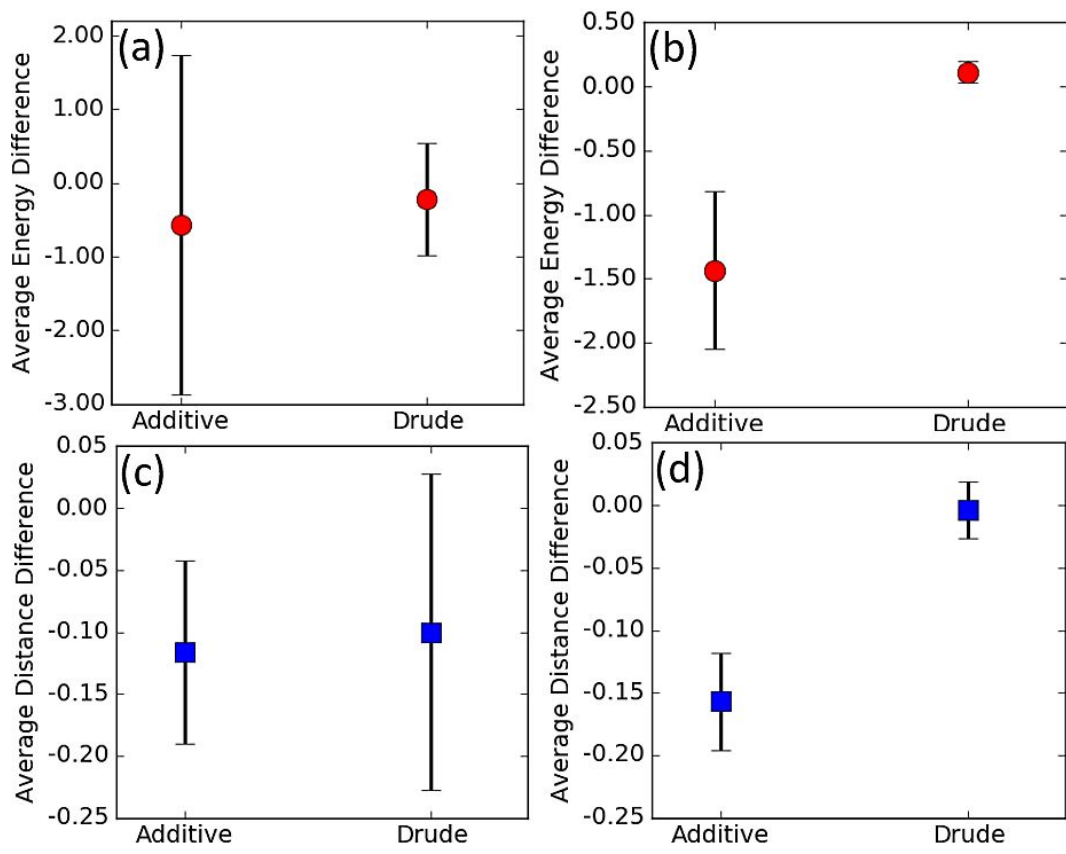

Figure S2. Figure showing average and standard deviation (represented as error bar) for MM -QM values of Solute-Water Pair Interaction energies (for model compound (a) M1, (c) M2); and Solute-Water Pair Interaction distances (for model compound (b) M1, (d) M2). 

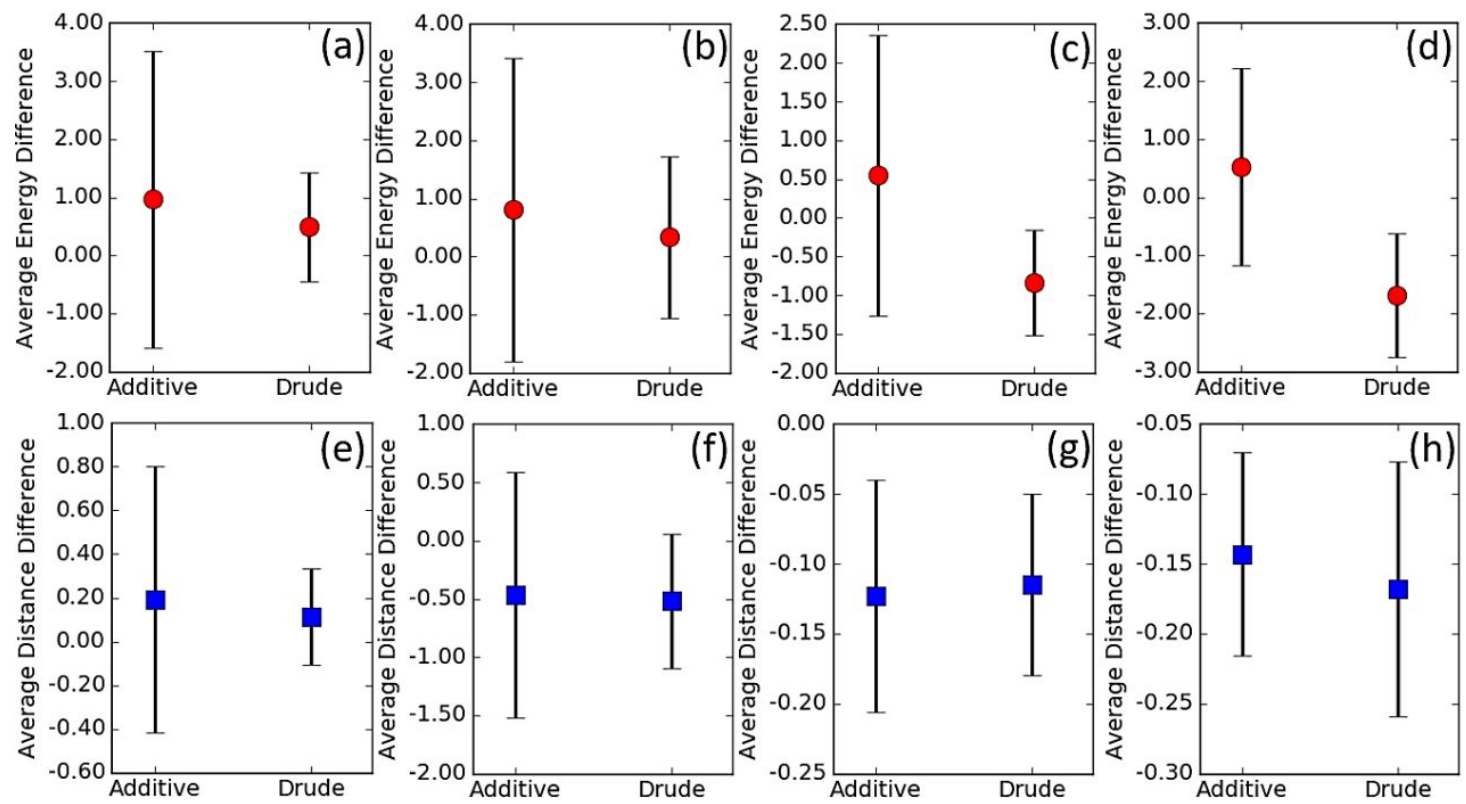

Figure S3. Figure showing average and standard deviation (represented as error bar) for MM

- QM values of Solute-Water Pair Interaction energies (for (a) for AGLCA, (b) BGLCA, (c) AIDOA, and (d) BIDOA); and Solute-Water Pair Interaction distances (for (e) AGLCA, (f) BGLCA, (g) AIDOA, and (h) BIDOA).
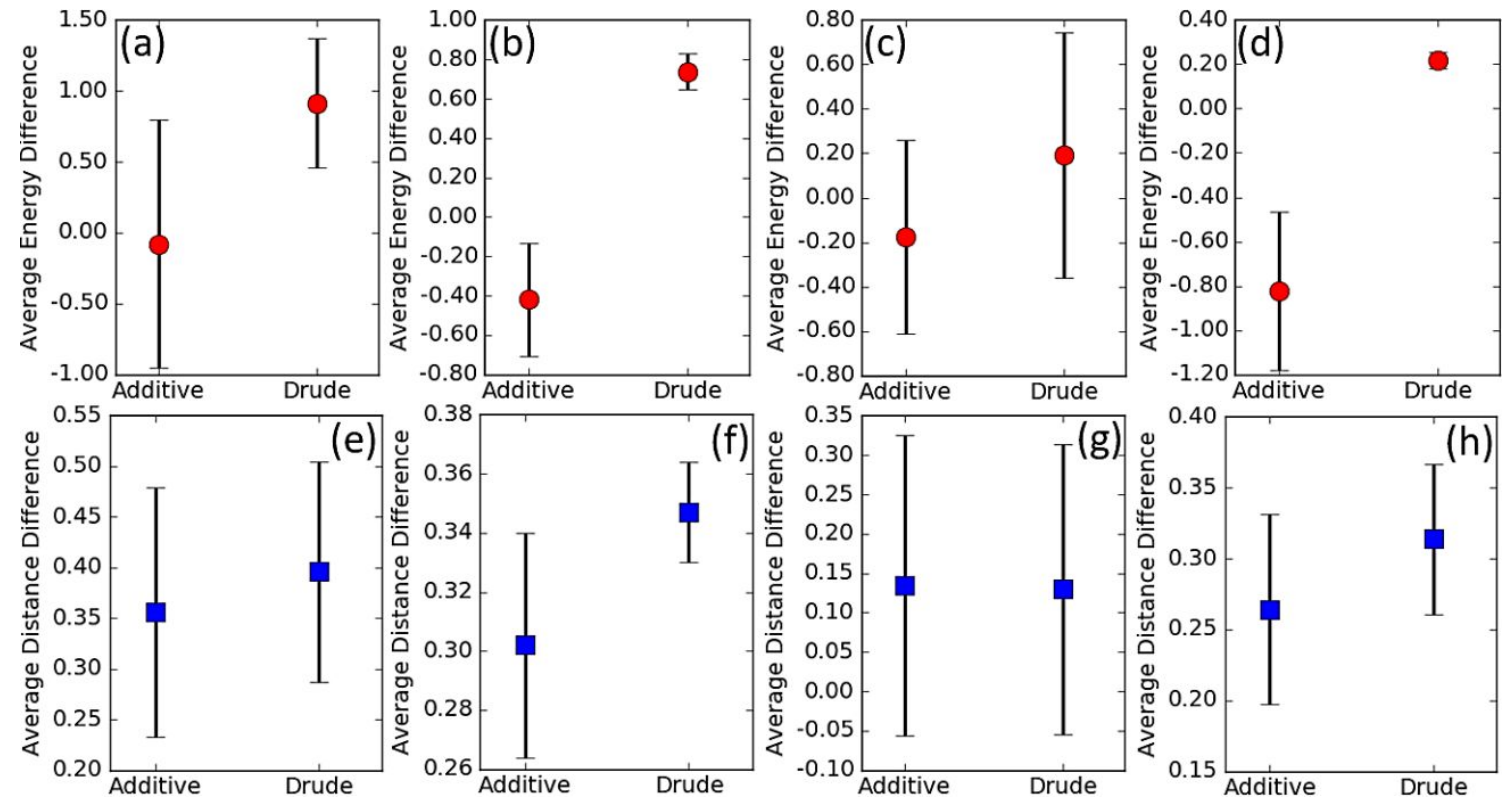

Figure S4. Figure showing average and standard deviation (represented as error bar) for MM -QM values of Solute-Water Pair Interaction energies (for (a) AGLCNA, (b) BGLCNA, (c) AGALNA, and (d) BGALNA); and Solute-Water Pair Interaction distances (for (e) AGLCNA, (f) BGLCNA, (g) AGALNA, and (h) BGALNA). 

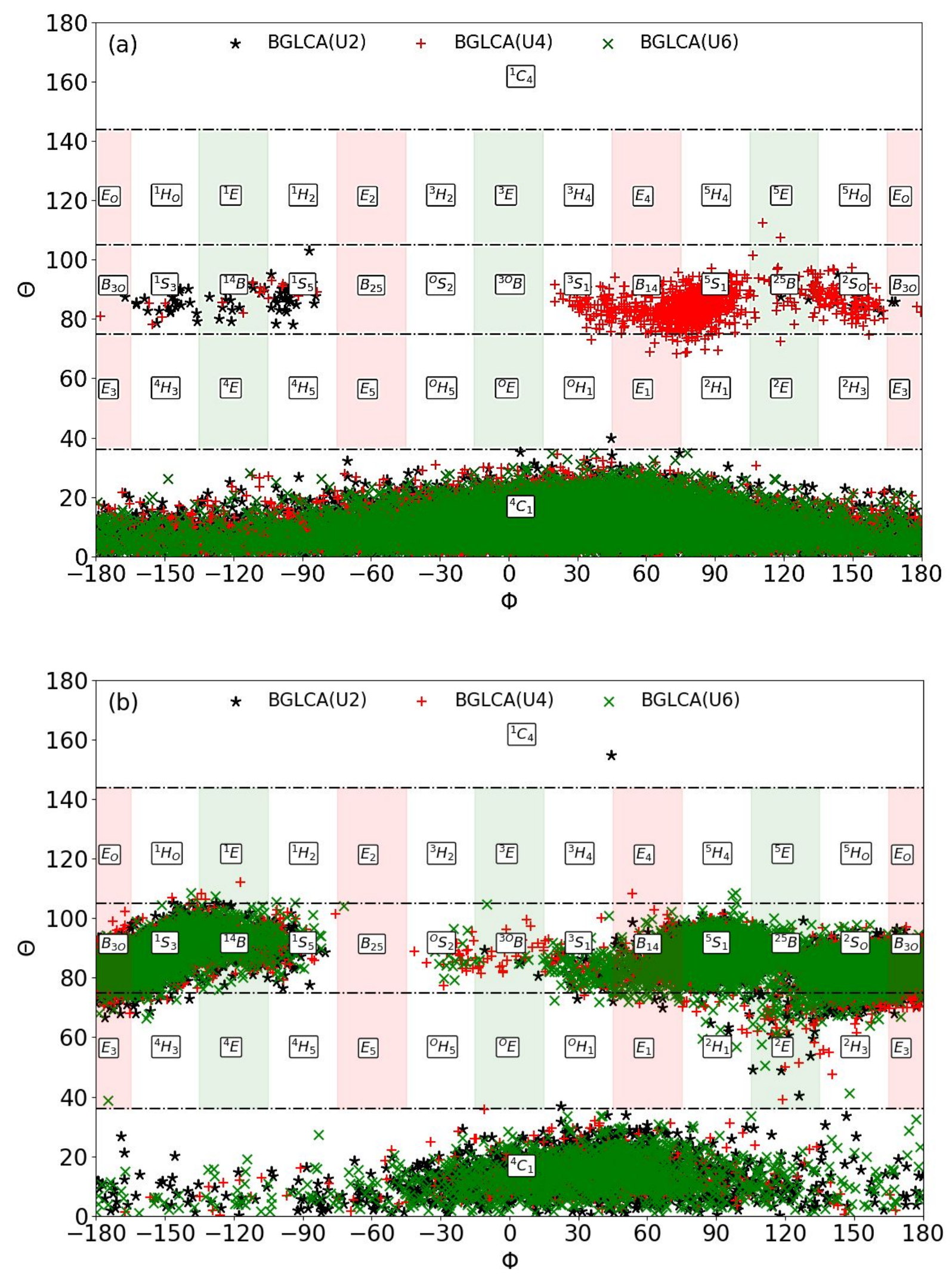

Figure S5. Conformations identified by standard mapping from IUPAC nomenclature to the Cremer-Pople puckering parameters ${ }^{12}$ for BGLCA from a 60 ns MD simulation of the unsulfated chondroitin NMR structure using additive (a) and Drude polarizable (b) force fields. 

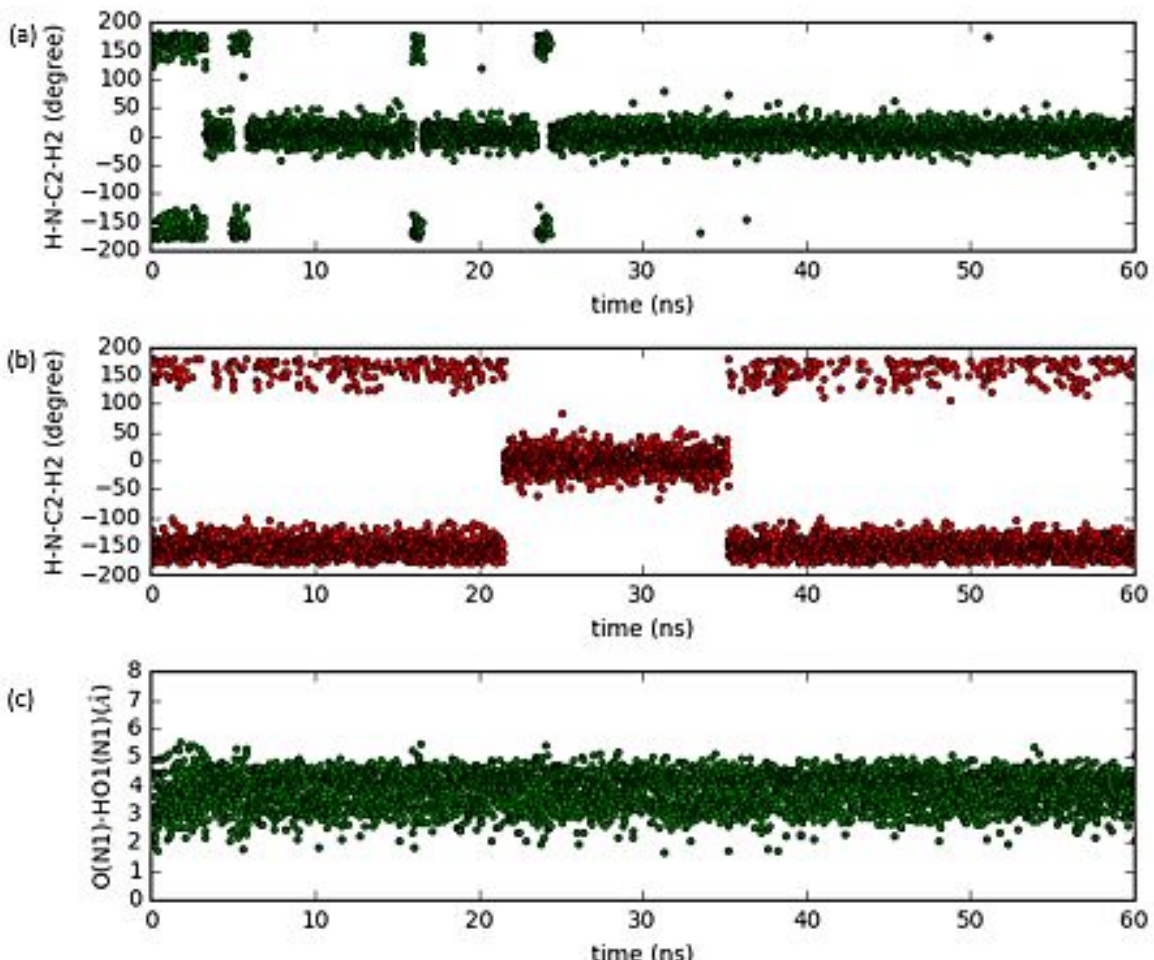

(d)

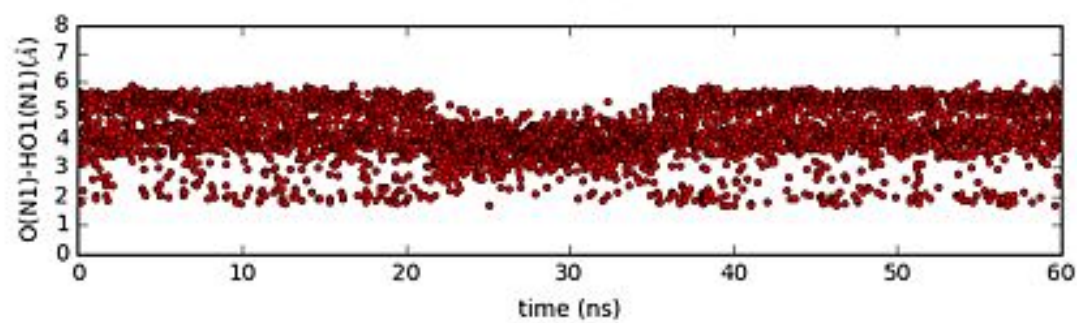

(e)

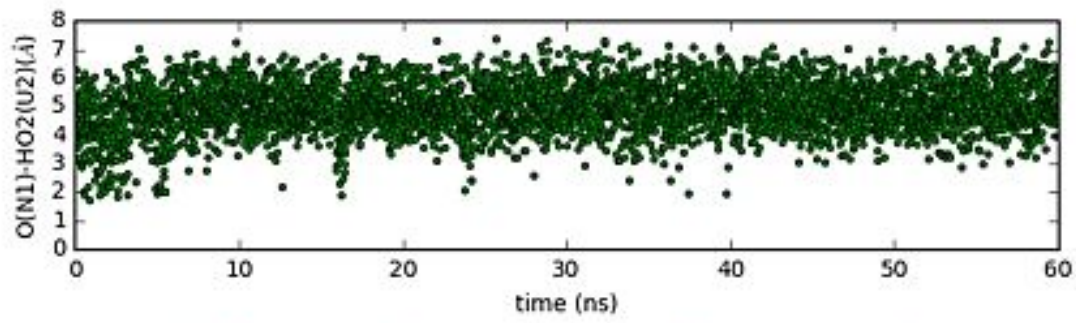

(f)

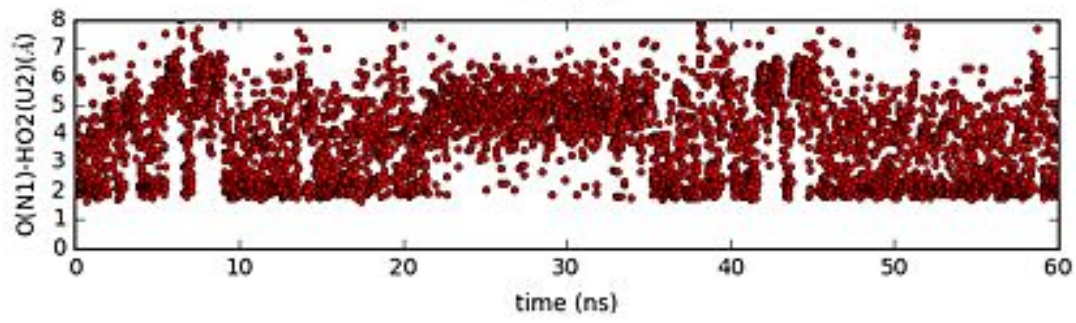

Figure S6. Conformational properties of the GlcNAc (N1) acetamido group from a 60 ns MD simulation of the unsulfated chondroitin NMR structure using additive (green) and Drude polarizable (red) force fields. Figure (a) and (b) show the H-N-C2-H2 dihedral (vs time) for additive and Drude, respectively; Figure (c) and (d) show the O(N1)-HO1(N1) intra-residue $\mathrm{H}$-bond distance (vs time) for additive and Drude, respectively; and Figure (e) and (f) show the $\mathrm{O}(\mathrm{N} 1)-\mathrm{HO} 2(\mathrm{U} 2)$ inter-residue H-bond distance (vs time) for additive and Drude, respectively. 

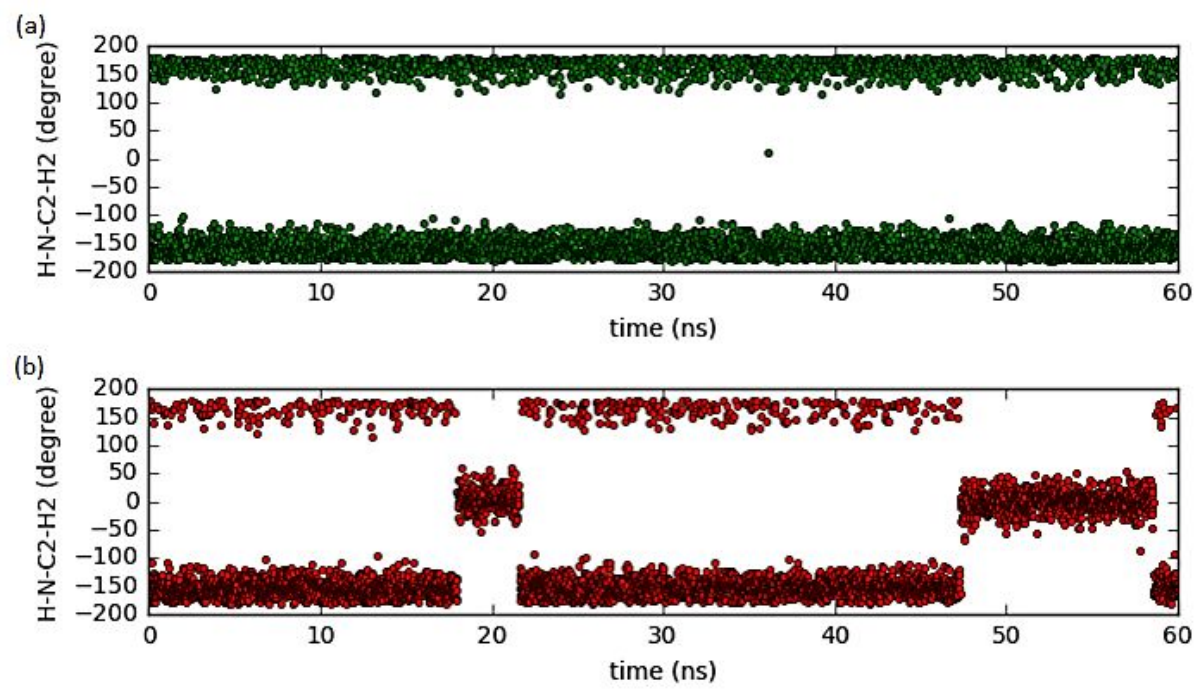

(c)

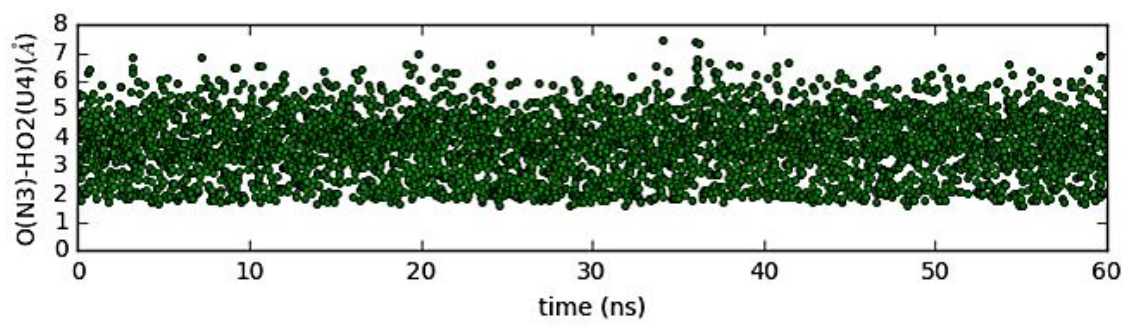

(d)

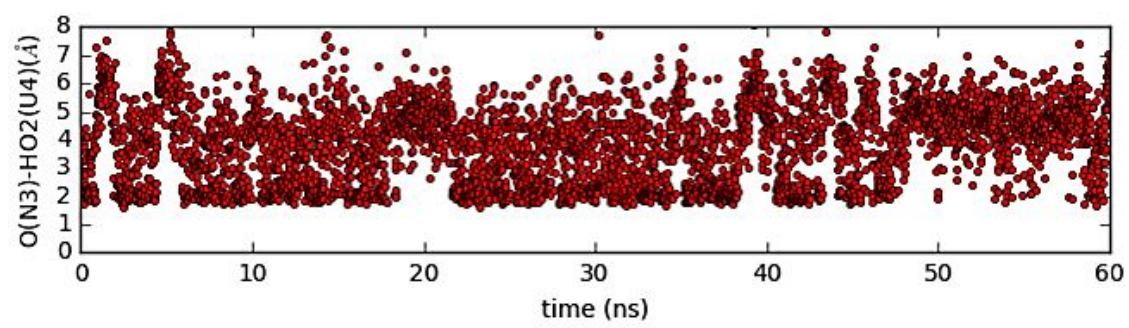

(e)

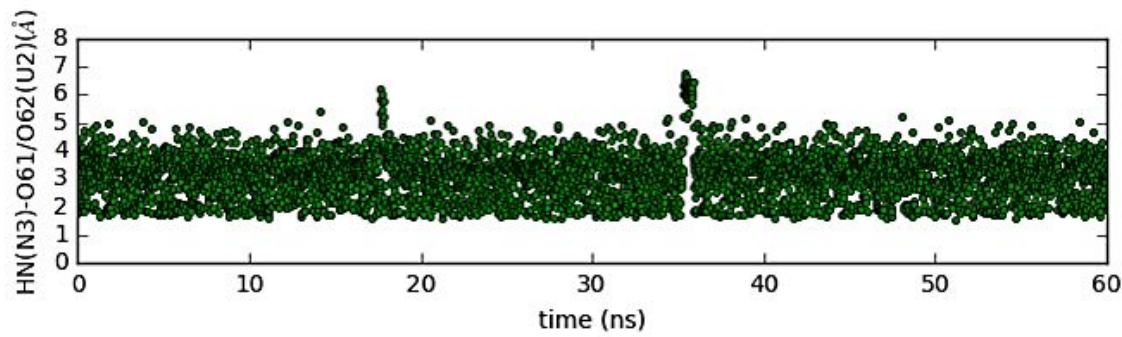

(f)

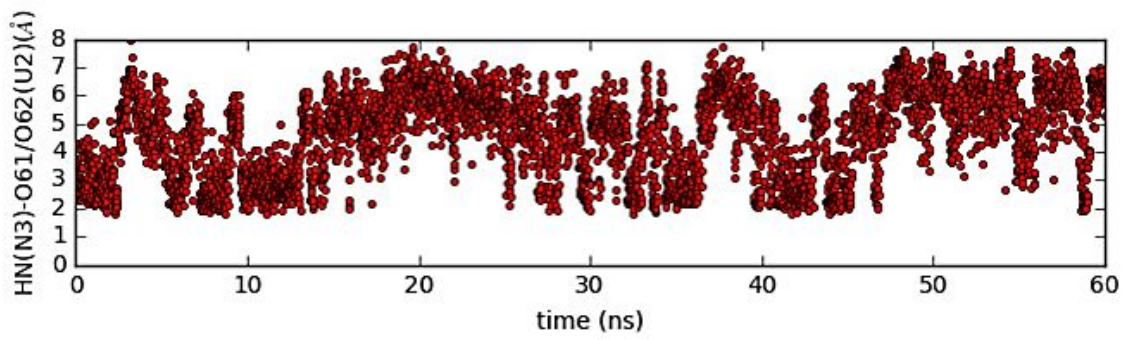

Figure S7. Conformational properties of the GlcNAc (N3) acetamido group from a 60 ns MD simulation of the unsulfated chondroitin NMR structure using additive (green) and Drude polarizable (red) force fields. Figure (a) and (b) show the H-N-C2-H2 dihedral (vs time) for additive and Drude, respectively; Figure (c) and (d) show the O(N3)-HO2(U4) inter-residue $\mathrm{H}$-bond distance (vs time) for additive and Drude, respectively; and Figure (e) and (f) show the $\mathrm{HN}(\mathrm{N} 3)-\mathrm{O} 61 / \mathrm{O} 62(\mathrm{U} 2)$ inter-residue H-bond distance (vs time) for additive and Drude, respectively. 

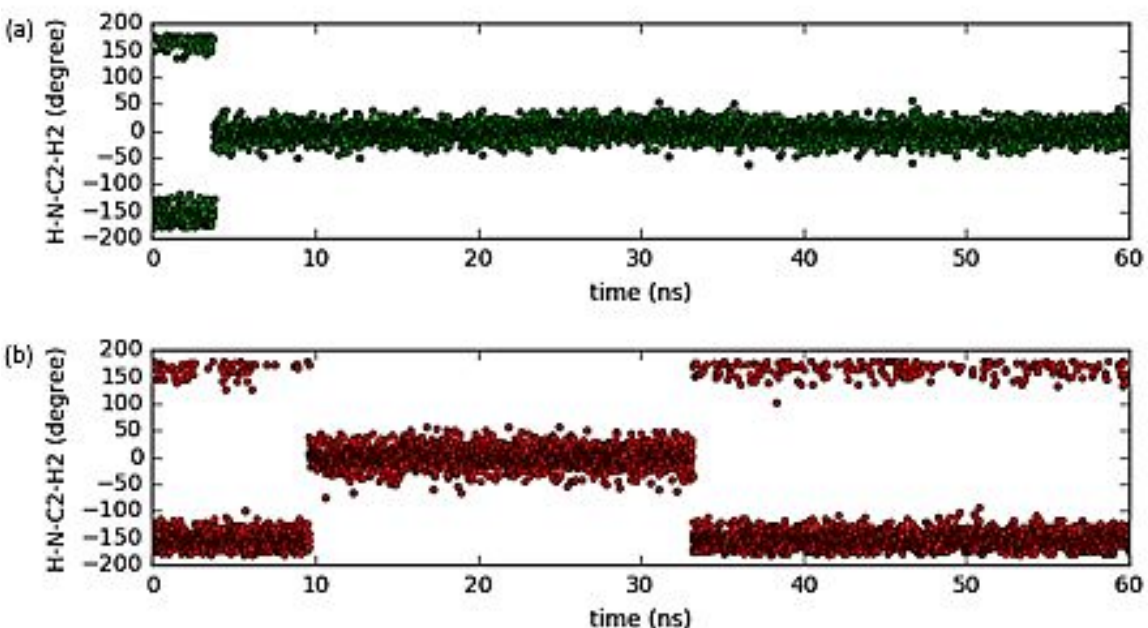

(c)

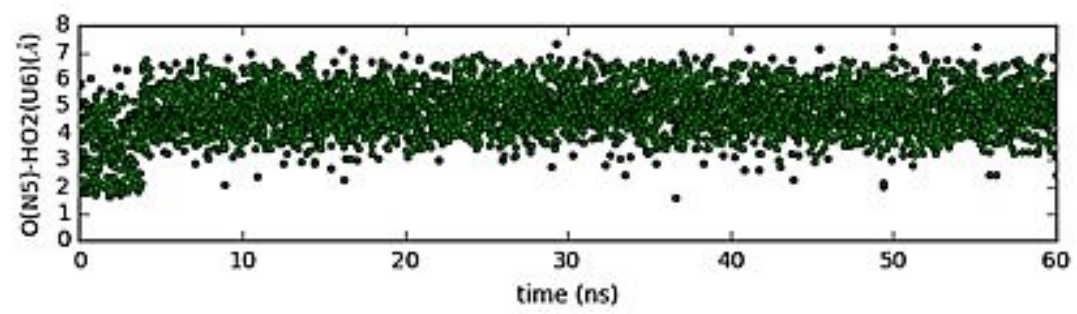

(d)

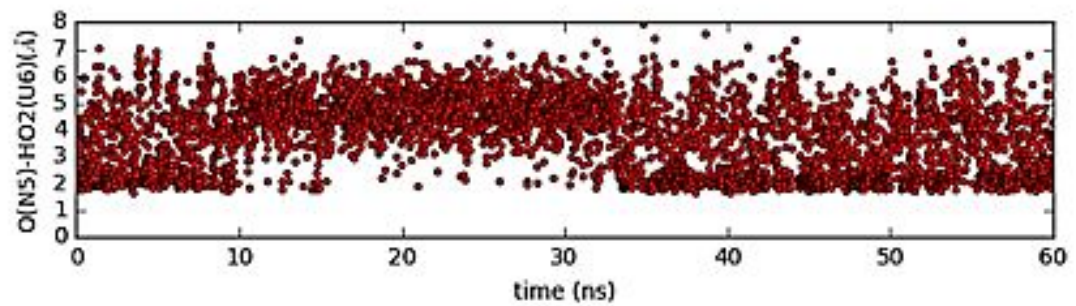

(e)
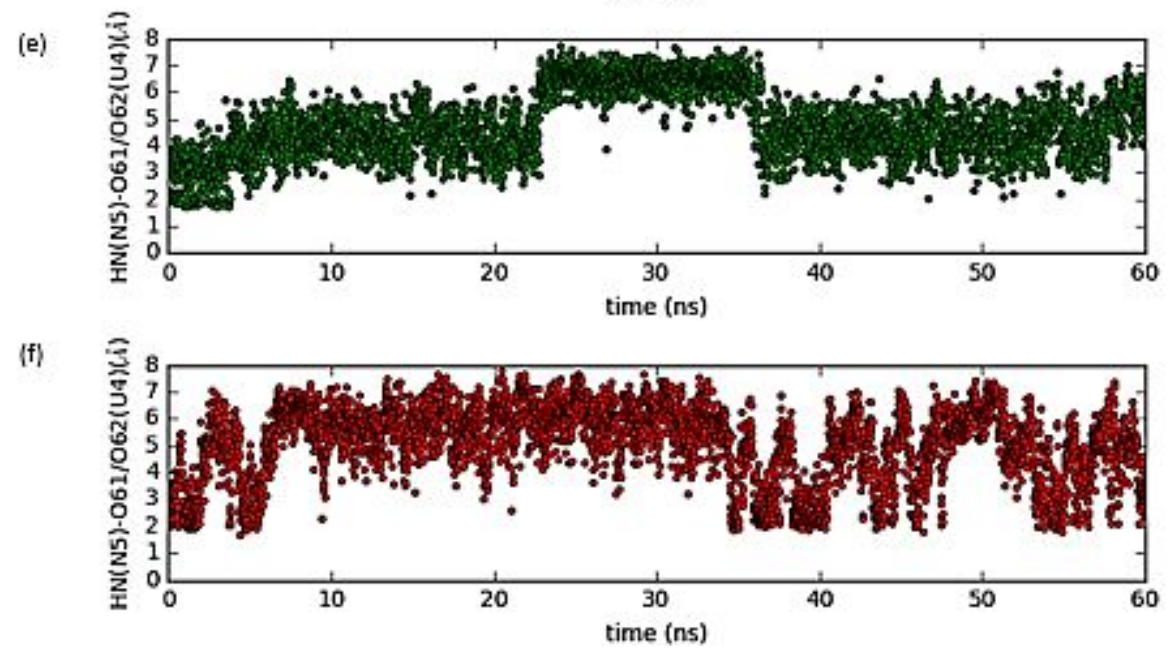

Figure S8. Conformational properties of the GlcNAc (N5) acetamido group from a 60 ns MD simulation of the unsulfated chondroitin NMR structure using additive (green) and Drude polarizable (red) force fields. Figure (a) and (b) showing the $\mathrm{H}-\mathrm{N}-\mathrm{C} 2-\mathrm{H} 2$ dihedral (vs time) for additive and Drude, respectively; Figure (c) and (d) showing the O(N5)-HO2(U6) interresidue H-bond distance (vs time) for additive and Drude, respectively; and Figure (e) and (f) showing the HN(N5)-O61/O62(U4) inter-residue H-bond distance (vs time) for additive and Drude, respectively. 


\section{References}

1. Sattelle, B. M.; Shakeri, J.; Roberts, I. S.; Almond, A., A 3D-structural model of unsulfated chondroitin from high-field NMR: 4-sulfation has little effect on backbone conformation. Carbohydrate research 2010, 345 (2), 291-302.

2. Jorgensen, W. L.; Chandrasekhar, J.; Madura, J. D.; Impey, R. W.; Klein, M. L., Comparison of simple potential functions for simulating liquid water. The Journal of chemical physics 1983, 79 (2), 926-935.

3. Suh, D.; Radak, B. K.; Chipot, C.; Roux, B., Enhanced configurational sampling with hybrid non-equilibrium molecular dynamics-Monte Carlo propagator. The Journal of chemical physics 2018, 148 (1), 014101.

4. Phillips, J. C.; Braun, R.; Wang, W.; Gumbart, J.; Tajkhorshid, E.; Villa, E.; Chipot, C.; Skeel, R. D.; Kale, L.; Schulten, K., Scalable molecular dynamics with NAMD. Journal of computational chemistry 2005, 26 (16), 1781-1802.

5. Darden, T.; York, D.; Pedersen, L., Particle mesh Ewald: An N. $\log (\mathrm{N})$ method for Ewald sums in large systems. The Journal of chemical physics 1993, 98 (12), 1008910092.

6. Feller, S. E.; Zhang, Y.; Pastor, R. W.; Brooks, B. R., Constant pressure molecular dynamics simulation: the Langevin piston method. The Journal of chemical physics 1995, 103 (11), 4613-4621.

7. Miyamoto, S.; Kollman, P. A., Settle: An analytical version of the SHAKE and RATTLE algorithm for rigid water models. Journal of computational chemistry 1992, 13 (8), 952-962.

8. Ryckaert, J.-P.; Ciccotti, G.; Berendsen, H. J., Numerical integration of the cartesian equations of motion of a system with constraints: molecular dynamics of nalkanes. Journal of Computational Physics 1977, 23 (3), 327-341.

9. Lamoureux, G.; Harder, E.; Vorobyov, I. V.; Roux, B.; MacKerell Jr, A. D., A polarizable model of water for molecular dynamics simulations of biomolecules. Chemical Physics Letters 2006, 418 (1-3), 245-249.

10. Lamoureux, G.; Roux, B. t., Modeling induced polarization with classical Drude oscillators: Theory and molecular dynamics simulation algorithm. The Journal of chemical physics 2003, 119 (6), 3025-3039.

11. Pulay, P.; Fogarasi, G.; Pang, F.; Boggs, J. E., Systematic ab initio gradient calculation of molecular geometries, force constants, and dipole moment derivatives. Journal of the American Chemical Society 1979, 101 (10), 2550-2560.

12. Cremer, D. t.; Pople, J., General definition of ring puckering coordinates. Journal of the American Chemical Society 1975, 97 (6), 1354-1358. 
